\title{
A UNIFIED SEPARATION THEOREM FOR CLOSED SETS IN A BANACH SPACE AND OPTIMALITY CONDITIONS FOR VECTOR OPTIMIZATION*
}

\author{
XI YIN ZHENG ${ }^{\dagger}$ AND KUNG FU NG ${ }^{\ddagger}$
}

\begin{abstract}
Using the technique of variational analysis and in terms of normal cones, we establish unified separation results for finitely many closed (not necessarily convex) sets in Banach spaces, which not only cover the existing nonconvex separation results and a classical convex separation theorem, but also recapture the approximate projection theorem. With help of the separation result for closed sets, we provide necessary and sufficient conditions for approximate Pareto solutions of constrained vector optimization problems. In particular, we extend some basic optimality results for approximate solutions of numerical optimization problems to the vector optimization setting.
\end{abstract}

Key words. normal cone, separation theorem, vector optimization, approximate Pareto solution

AMS subject classifications. 49J52, 90C25, 90C29

DOI. $10.1137 / 100811155$

1. Introduction. The separation theorems for convex sets play a key role in functional analysis and optimization theory. The most well-known and useful version of these theorems is probably the following: if $A_{1}$ and $A_{2}$ are disjoint closed convex sets in $X$ with one of them being compact, then there exists a continuous linear functional $x^{*}$ on $X$ such that

$$
\inf _{x \in A_{2}}\left\langle x^{*}, x\right\rangle>\sup _{x \in A_{1}}\left\langle x^{*}, x\right\rangle
$$

where $X$ is a Banach space (or more generally, a locally convex topological vector space). In order to focus on the main issues and also for the simplicity of presentation, we assume throughout that $X$ is a Banach space (we shall explicitly make clear if $X$ is required to satisfy additional assumptions, such as that $X$ is an Asplund space). In recent years, a lot of attention has been directed to studying the more general case that $A_{1}, A_{2}$ are closed (not necessarily convex) subsets of $X$ (cf. [14], [22], [23], and references therein). In an Asplund space and in terms of Fréchet normal cone, Mordukhovich and Shao [15] first established the extremal principle for two closed sets with an extremal point (a special common point of these two sets). In some sense, this extremal principle can be regarded as a kind of fuzzy separation theorem for two nonconvex closed subsets. Further, Mordukhovich, Treiman, and Zhu [17] introduced the extremal point concept for finitely many closed sets and established the extremal principle for finitely many closed sets. At this point, let us define the so-called nonintersect index $\gamma\left(A_{1}, \ldots, A_{n}\right)$ by

*Received by the editors October 8, 2010; accepted for publication (in revised form) June 27, 2011; published electronically September 15, 2011. This research was supported by an earmarked grant from the Research Grant Council of Hong Kong (GRF) and the National Natural Science Foundation of the People's Republic of China (grant 11061038).

http://www.siam.org/journals/siopt/21-3/81115.html

'Department of Mathematics, Yunnan University, Kunming 650091, People's Republic of China (xyzheng@ynu.edu.cn).

${ }^{\ddagger}$ Department of Mathematics (and IMS), The Chinese University of Hong Kong, Shatin, New Territory, Hong Kong (kfng@math.cuhk.edu.hk). 


$$
\gamma\left(A_{1}, \ldots, A_{n}\right):=\inf \left\{\sum_{i=1}^{n-1}\left\|a_{i}-a_{n}\right\|: a_{i} \in A_{i}, i=1, \ldots, n\right\} .
$$

Note that $\gamma\left(A_{1}, \ldots, A_{n}\right)=0$ if $\bigcap_{i=1}^{n} A_{i} \neq \varnothing$ and that for any $\varepsilon>0$, there exists $a_{i} \in A_{i}$ $(1 \leq i \leq n)$ such that

$$
\sum_{i=1}^{n-1}\left\|a_{i}-a_{n}\right\|<\gamma\left(A_{1}, \ldots, A_{n}\right)+\varepsilon
$$

Improving the extremal principles by Mordukhovich et al., the author [29] established the following result.

Theorem A. Consider closed sets $A_{1}, \ldots, A_{n}$ of a Banach (resp., Asplund) space $X$ such that $\bigcap_{i=1}^{n} A_{i}=\varnothing$. Let $\varepsilon>0$ and $a_{i} \in A_{i}(1 \leq i \leq n)$ satisfy (1.1). Then, for any $\lambda>0$, there exist $\bar{a}_{i} \in A_{i}$ and $a_{i}^{*} \in X^{*}$ such that

(i) $\sum_{i=1}^{n}\left\|\bar{a}_{i}-a_{i}\right\|<\lambda, \max _{1 \leq i \leq n-1}\left\|a_{i}^{*}\right\|=1$, and $\sum_{i=1}^{n} a_{i}^{*}=0$;

(ii) $a_{i}^{*} \in N_{c}\left(A_{i}, \bar{a}_{i}\right)+\frac{\varepsilon}{\lambda} B_{X^{*}}\left(\right.$ resp., $\left.a_{i}^{*} \in \hat{N}\left(A_{i}, \bar{a}_{i}\right)+\frac{\varepsilon}{\lambda} B_{X^{*}}\right), i=1, \ldots, n$, where $N_{c}\left(A_{i}, \bar{a}_{i}\right)$ and $\hat{N}\left(A_{i}, \bar{a}_{i}\right)$ denote, respectively, the Clarke and Fréchet normal cones (see section 2 for their definitions).

Unfortunately, even in the case when $n=2, A_{1}=\{x\}$, and $A_{2}$ is convex (and closed) such that $x \notin A_{2}$, this theorem and all other existing fuzzy separation results for general closed sets cannot recapture the classical separation theorem stated at the beginning of this section. On the other hand, by the approximate projection theorem for a closed set (proved by the authors [30] and [12]), for any $\eta \in(0,1)$, there exist $\bar{a}_{2} \in$ $A_{2}$ and $-a_{2}^{*} \in N_{c}\left(A_{2}, \bar{a}_{2}\right)$ such that $\left\|a_{2}^{*}\right\|=1$ and

$$
\eta\left\|x-\bar{a}_{2}\right\| \leq\left\langle a_{2}^{*}, \bar{a}_{2}-x\right\rangle
$$

Clearly, (1.2) does imply that $A_{1}=\{x\}$ and $A_{2}$ can be separated (in the usual sense) if $A_{2}$ is convex. From the theoretical viewpoint as well as for applications, it is important and interesting to have a new kind of fuzzy separation theorem that can result in existing fuzzy separation theorems and classical convex separation results. It is one of our aims to establish such fuzzy separation results for closed sets.

Vector optimization relates to functional analysis and mathematical programming and has been found to play many important roles in economics theory, engineering design, management science, and so on. In recent years, the study of vector optimization has received increasing attention in the literature (see [7], [10], [13], and references therein). Another aim of this paper is to study constrained vector optimization problems and thereby improve and extend some well-known results on numerical optimization. Many authors (cf. [14], [20], [23], and references therein) studied a numerical optimization problem with a constraint defined by finitely many inequalities and equalities. Most of the earlier authors provide necessary/sufficient conditions for a feasible point to be a solution, and their studies are based on the assumption that the problem concerned does have a (local or global) solution. On one hand, this assumption is too restrictive in some contexts, while, on the other hand, we note a well-known fact: if a function $\phi_{0}: X \rightarrow \mathbb{R}$ is smooth and bounded below, then for any $\varepsilon>0$ there exists $x_{\varepsilon} \in X$ such that

$$
\phi_{0}\left(x_{\varepsilon}\right)<\inf _{x \in X} \phi_{0}(x)+\varepsilon \quad \text { and } \quad\left\|\phi_{0}^{\prime}\left(x_{\varepsilon}\right)\right\|<\varepsilon .
$$

Copyright ( by SIAM. Unauthorized reproduction of this article is prohibited. 
Without the smoothness assumption, in a geometric constraint case, Chou, $\mathrm{Ng}$, and Pang [4] proved the following result: if $\phi_{0}$ is Lipschitz and bounded below on a closed subset $A$ of $X$, then for any $\varepsilon>0$ there exists $x_{\varepsilon} \in A$ such that

$$
\phi\left(x_{\varepsilon}\right)<\inf _{x \in A} \phi_{0}(x)+\varepsilon \quad \text { and } \quad d\left(0, \partial \phi_{0}\left(x_{\varepsilon}\right)+N(A, \varepsilon)\right)<\varepsilon .
$$

Mordukhovich and Wang [18] studied suboptimality conditions for approximate solutions for a numerical constraint optimization problem in infinite dimensional Asplund spaces. In particular, they established the Lagrange rule of an approximate solution for such a problem in terms of subdifferentials. With the help of the separation theorem for finitely many closed sets, this and other related results, as well as the result of $\mathrm{Chou}, \mathrm{Ng}$, and Pang mentioned above, are extended in section 4 for vector optimization problems.

2. Preliminaries. For convenience of the readers, this section recalls some known notions and results in variational analysis, which will be used in our later analysis (see [14], [22], [23] for more details).

We use $B_{X}$ and $\Sigma_{X}$ to denote the unit ball and unit sphere of $X$, respectively, and $B(x, r)$ denotes the open ball with center $a$ and radius $r$. Let $A$ be a closed subset of $X$ and $a$ be a point in $A$. We denote by $T_{c}(A, a)$ and $T(A, a)$ the Clarke tangent cone and the contingent (Bouligand) cone of $A$ at $a$, respectively; that is,

$$
T_{c}(A, a):=\left\{v \in X: \forall a_{n} \stackrel{A}{\rightarrow} a \text { and } \forall t_{n} \rightarrow 0^{+} \exists v_{n} \rightarrow v \text { s.t. } a_{n}+t_{n} v_{n} \in A \forall n \in \mathbb{N}\right\}
$$

and

$$
T(A, a):=\left\{v \in X: \exists t_{n} \rightarrow 0^{+} \text {and } v_{n} \rightarrow v \text { s.t. } a+t_{n} v_{n} \in A \forall n \in \mathbb{N}\right\} .
$$

The Clarke normal cone $N_{c}(A, a)$ of $A$ at $a$ is defined by

$$
N_{c}(A, a):=\left\{x^{*} \in X^{*} \mid\left\langle x^{*}, h\right\rangle \leq 0 \forall h \in T_{c}(A, a)\right\} .
$$

For $\varepsilon \geq 0$ and $a \in A$, the nonempty set

$$
\hat{N}_{\varepsilon}(A, a):=\left\{x^{*} \in X^{*} \mid \lim _{\substack{A \\ x \rightarrow a}} \sup \frac{\left\langle x^{*}, x-a\right\rangle}{\|x-a\|} \leq \varepsilon\right\}
$$

is called the set of Fréchet $\varepsilon$-normals of $A$ at $a$, where $x \stackrel{A}{\rightarrow} a$ means $x \rightarrow a$ and $x \in A$. When $\varepsilon=0, \hat{N}_{\varepsilon}(A, a)$ is a convex cone which is called the Fréchet normal cone of $A$ at $a$ and is denoted by $\hat{N}(A, a)$. The Mordukhovich (limiting) normal cone $N(A, a)$ of $A$ at $a$ is defined by

$$
N(A, a):=\left\{x^{*} \in X^{*}: \exists \varepsilon_{n} \rightarrow 0^{+}, a_{n} \stackrel{A}{\rightarrow} a \text { and } x_{n}^{*} \stackrel{w^{*}}{\rightarrow} x^{*} \text { s.t. } x_{n}^{*} \in \hat{N}_{\varepsilon_{n}}\left(A, a_{n}\right) \forall n \in \mathbb{N}\right\} .
$$

It is known (cf. [14], [23]) that

$$
\hat{N}(A, a) \subset N(A, a) \subset N_{c}(A, a) .
$$

Mordukhivich and Shao [16] proved that if $X$ is an Asplund space, then

$$
N_{c}(A, a)=\operatorname{cl}^{*}(\operatorname{co}(N(A, a))) \quad \text { and } \quad N(A, a)=\lim _{\substack{A \\ x \rightarrow a}} \sup \hat{N}(A, x),
$$

Copyright ( by SIAM. Unauthorized reproduction of this article is prohibited. 
where $\mathrm{cl}^{*}$ denotes the weak ${ }^{*}$ closure. It is well known that if $A$ is a convex set, then $T_{c}(A, a)=T(A, a)$ and

$$
N_{c}(A, a)=\hat{N}(A, a)=\left\{x^{*} \in X^{*} \mid\left\langle x^{*}, x\right\rangle \leq\left\langle x^{*}, a\right\rangle \forall x \in A\right\} .
$$

Let $\phi: X \rightarrow \mathbb{R} \cup\{+\infty\}$ be a proper lower semicontinuous function. The ClarkeRockafellar subdifferential $\partial_{c} \phi(x)$ of $\phi$ at $x \in \operatorname{dom}(\phi)$ is defined as

$$
\partial_{c} \phi(x):=\left\{x^{*} \in X^{*} \mid\left\langle x^{*}, h\right\rangle \leq \phi^{\uparrow}(x, h) \forall h \in X\right\},
$$

where

$$
\phi^{\uparrow}(x, h):=\lim _{\varepsilon \downarrow 0} \lim _{\substack{\phi \\ z \rightarrow x, t \downarrow 0}} \sup _{w \in h+\varepsilon B_{x}} \frac{\phi(z+\mathrm{tw})-\phi(z)}{t} .
$$

The Fréchet subdifferential of $\phi$ at $x \in \operatorname{dom}(\phi)$ is defined as

$$
\hat{\partial} \phi(x):=\left\{x^{*} \in X^{*} \mid \liminf _{z \rightarrow x} \frac{\phi(z)-\phi(x)-\left\langle x^{*}, z-x\right\rangle}{\|z-x\|} \geq 0\right\} .
$$

It is well known (cf. [14]) that

$$
\hat{\partial} \phi(x) \subset \partial_{c} \phi(x)
$$

and that if $\phi$ is convex, then

$$
\partial_{c} \phi(x)=\hat{\partial} \phi(x)=\left\{x^{*} \in X^{*} \mid\left\langle x^{*}, y-x\right\rangle \leq \phi(y)-\phi(x) \forall y \in X\right\} \quad \forall x \in \operatorname{dom}(\phi) .
$$

For a closed set $A$ in $X$, let $\delta_{A}$ denote the indicator function of $A$. It is known (see [14], [23]) that

$$
N_{c}(A, a)=\partial_{c} \delta_{A}(a), \hat{N}(A, a)=\hat{\partial} \delta_{A}(a) \quad \forall a \in A
$$

and

$$
\begin{gathered}
\partial_{c} \phi(x)=\left\{x^{*} \in X^{*} \mid\left(x^{*},-1\right) \in N_{c}(\operatorname{epi}(\phi),(x, \phi(x)))\right\} \quad \forall x \in \operatorname{dom}(\phi), \\
\hat{\partial} \phi(x)=\left\{x^{*} \in X^{*} \mid\left(x^{*},-1\right) \in \hat{N}(\operatorname{epi}(\phi),(x, \phi(x)))\right\} \quad \forall x \in \operatorname{dom}(\phi),
\end{gathered}
$$

where epi $(\phi):=\{(x, t) \in X \times \mathbb{R}: \phi(x) \leq t\}$.

We recall the following known subdifferential rules for the sum-function (cf. [14], [22], [23]), which plays a important role in our later analysis.

Lemma 2.1. Let $\phi_{1}, \phi_{2}: X \rightarrow \mathbb{R} \cup\{+\infty\}$ be proper lower semicontinuous functions. Let $x \in \operatorname{dom}\left(\phi_{1}\right) \cap \operatorname{dom}\left(\phi_{2}\right)$, and suppose that $\phi_{1}$ is locally Lipschitz around $x$. Then,

$$
\partial_{c}\left(\phi_{1}+\phi_{2}\right)(x) \subset \partial_{c} \phi_{1}(x)+\partial_{c} \phi_{2}(x) .
$$

If, in addition, $X$ is an Asplund space, then for any $x^{*} \in \hat{\partial}\left(\phi_{1}+\phi_{2}\right)(x)$ and any $\varepsilon>0$ there exist $x_{1}, x_{2} \in B(x, \varepsilon)$ such that $\left|\phi_{i}\left(x_{i}\right)-\phi_{i}(x)\right|<\varepsilon(i=1,2)$ and

$$
x^{*} \in \hat{\partial} \phi_{1}\left(x_{1}\right)+\hat{\partial} \phi_{2}\left(x_{2}\right)+\varepsilon B_{X^{*}} .
$$

Copyright ( $\odot$ by SIAM. Unauthorized reproduction of this article is prohibited. 
For a multifunction $F$ between Banach spaces $X$ and $Y$, we use $\operatorname{Gr}(F)$ to denote its graph, and say that it is closed (resp., convex) if $\operatorname{Gr}(F)$ is a closed (resp., convex) subset of $X \times Y$. Recall (cf. [2], [9]) that $F$ is pseudo-Lipschitz at $(\bar{x}, \bar{y}) \in \operatorname{Gr}(F)$ if there exist $L$, $r_{1}, r_{2} \in(0,+\infty)$ such that

$$
F\left(x_{1}\right) \cap B\left(\bar{y}, r_{1}\right) \subset F\left(x_{2}\right)+\left\|x_{1}-x_{2}\right\| L B_{Y} \quad \forall x_{1}, x_{2} \in B\left(\bar{x}, r_{2}\right) .
$$

For $x \in X$ and $y \in F(x)$, let $\hat{D}^{*} F(x, y)$ and $D_{c}^{*} F(x, y): Y^{*} \rightrightarrows X^{*}$ denote the coderivatives of $F$ at $(x, y)$ with respect to the Fréchet and Clarke normal cones, respectively; that is,

$$
\hat{D}^{*} F(x, y)\left(y^{*}\right):=\left\{x^{*} \in X^{*}:\left(x^{*},-y^{*}\right) \in \hat{N}(\operatorname{Gr}(F),(x, y))\right\} \quad \forall y^{*} \in Y^{*}
$$

and

$$
D_{c}^{*} F(x, y)\left(y^{*}\right):=\left\{x^{*} \in X^{*}:\left(x^{*},-y^{*}\right) \in N_{c}(\operatorname{Gr}(F),(x, y))\right\} \quad \forall y^{*} \in Y^{*} .
$$

3. Fuzzy separation results. In this section, we establish fuzzy separation results for finitely many closed sets, which not only unifies the convex separation theorem mentioned in section 1 and the existing nonconvex separation results, but also recaptures the approximate projection theorem proved in [30] and [12].

Let $1 \leq p \leq+\infty$ and $\gamma_{p}\left(A_{1}, \ldots, A_{n}\right)$ denote the ( $p$-weighted) nonintersect index of finitely many closed subsets $A_{1}, \ldots, A_{n}$ of a Banach space $X$, which is defined by

$$
\gamma_{p}\left(A_{1}, \ldots, A_{n}\right):=\inf \left\{\left(\sum_{i=1}^{n-1}\left\|x_{i}-x_{n}\right\|^{p}\right)^{\frac{1}{p}}: x_{i} \in A_{i}, i=1, \ldots, n\right\}
$$

where $\left(\sum_{i=1}^{n-1}\left\|x_{i}-x_{n}\right\|^{p}\right)^{\frac{1}{p}}$ is understood as $\max _{0 \leq i \leq n}\left\|x_{i}-x_{n}\right\|$ when $p=+\infty$.

For a point $e$ and two subsets $S_{1}$ and $S_{2}$ of a Banach space, let

$$
d\left(S_{1}, S_{2}\right):=\inf \left\{\|u-v\|: u \in S_{1} \text { and } v \in S_{2}\right\} \quad \text { and } \quad d\left(e, S_{2}\right):=d\left(\{e\}, S_{2}\right) .
$$

Theorem 3.1. Let $A_{1}, \ldots, A_{n}$ be closed nonempty subsets of $X$ such that $\bigcap_{i=1}^{n} A_{i}=\varnothing$. Let $1 \leq p, q \leq+\infty$ with $\frac{1}{p}+\frac{1}{q}=1, \varepsilon>0$, and $a_{i} \in A_{i}(1 \leq i \leq n)$ be such that

$$
\left(\sum_{i=1}^{n-1}\left\|a_{i}-a_{n}\right\|^{p}\right)^{\frac{1}{p}}<\gamma_{p}\left(A_{1}, \ldots, A_{n}\right)+\varepsilon
$$

Then, for any $\lambda>0$, there exist $\bar{a}_{i} \in A_{i}$ and $a_{i}^{*} \in X^{*}$ with the following properties:

(i) $\left(\sum_{i=1}^{n}\left\|\bar{a}_{i}-a_{i}\right\|^{p}\right)^{\frac{1}{p}}<\lambda$.

(ii) $\left(\sum_{i=1}^{n-1}\left\|a_{i}^{*}\right\|^{q}\right)^{\frac{1}{q}}=1, \sum_{i=1}^{n} a_{i}^{*}=0$, and $\left(\sum_{i=1}^{n} d\left(a_{i}^{*}, N_{c}\left(A_{i}, \bar{a}_{i}\right)\right)^{q}\right)^{\frac{1}{q}}<\frac{\varepsilon}{\lambda}$.

(iii) $\left(\sum_{i=1}^{n-1}\left\|\bar{a}_{n}-\bar{a}_{i}\right\|^{p}\right)^{\frac{1}{p}}=\sum_{i=1}^{n-1}\left\langle a_{i}^{*}, \bar{a}_{n}-\bar{a}_{i}\right\rangle$.

Proof. Define $\phi: X^{n} \rightarrow \mathbb{R} \cup\{+\infty\}$ as follows:

$$
\phi\left(x_{1}, \ldots, x_{n}\right):=\left(\sum_{i=1}^{n-1}\left\|x_{i}-x_{n}\right\|^{p}\right)^{\frac{1}{p}}+\delta_{A_{1} \times \cdots \times A_{n}}\left(x_{1}, \ldots, x_{n}\right)
$$

for all $\left(x_{1}, \ldots, x_{n}\right) \in X^{n}$, where $X^{n}$ is equipped with the norm

Copyright ( by SIAM. Unauthorized reproduction of this article is prohibited. 


$$
\left\|\left(x_{1}, \ldots, x_{n}\right)\right\|=\left(\sum_{i=1}^{n}\left\|x_{i}\right\|^{p}\right)^{\frac{1}{p}} \quad \forall\left(x_{1}, \ldots, x_{n}\right) \in X^{n} .
$$

Then

$$
\gamma_{p}\left(A_{1}, \ldots, A_{n}\right)=\inf \left\{\phi\left(x_{1}, \ldots, x_{n}\right):\left(x_{1}, \ldots, x_{n}\right) \in X^{n}\right\},
$$

and (by (3.1)) there exists $\varepsilon^{\prime} \in(0, \varepsilon)$ such that

$$
\phi\left(a_{1}, \ldots, a_{n}\right)<\inf \left\{\phi\left(x_{1}, \ldots, x_{n}\right):\left(x_{1}, \ldots, x_{n}\right) \in X^{n}\right\}+\varepsilon^{\prime} .
$$

Since $\phi$ is a lower semicontinuous function on the Banach space $X^{n}$, it follows from the Ekeland variational principle (cf. [14, Theorem 2.26]) that there exists $\left(\bar{a}_{1}, \ldots, \bar{a}_{n}\right) \in$ $X^{n}$ such that (i) holds and

$$
\phi\left(\bar{a}_{1}, \ldots, \bar{a}_{n}\right) \leq \phi\left(x_{1}, \ldots, x_{n}\right)+\frac{\varepsilon^{\prime}}{\lambda}\left(\sum_{i=1}^{n}\left\|x_{i}-\bar{a}_{i}\right\|^{p}\right)^{\frac{1}{p}} \quad \forall\left(x_{1}, \ldots, x_{n}\right) \in X^{n} .
$$

Hence $\phi\left(\bar{a}_{1}, \ldots, \bar{a}_{n}\right)<+\infty$ and so $\bar{a}_{i} \in A_{i}$ for each $i$. Noting that $\bigcap_{i=1}^{n} A_{i}=\varnothing$, it follows that

$$
\left(\bar{a}_{1}-\bar{a}_{n}, \ldots, \bar{a}_{n-1}-\bar{a}_{n}\right) \neq(0, \ldots, 0) .
$$

For each $\left(x_{1}, \ldots, x_{n}\right) \in X^{n}$, let

$$
f\left(x_{1}, \ldots, x_{n}\right):=\left(\sum_{i=1}^{n-1}\left\|x_{i}-x_{n}\right\|^{p}\right)^{\frac{1}{p}}+\frac{\varepsilon^{\prime}}{\lambda}\left(\sum_{i=1}^{n}\left\|x_{i}-\bar{a}_{i}\right\|^{p}\right)^{\frac{1}{p}} .
$$

Then $f$ is a continuous convex function on $X^{n}$, and (3.2) means that $f$ attains its minimum over $A_{1} \times \cdots \times A_{n}$ at $\left(\bar{a}_{1}, \ldots, \bar{a}_{n}\right)$. Hence $0 \in \partial_{c}\left(f+\delta_{A_{1} \times \cdots \times A_{n}}\right)\left(\bar{a}_{1}, \ldots, \bar{a}_{n}\right)$. This and Lemma 2.1 imply that

$$
0 \in \partial g\left(\bar{a}_{1}, \ldots, \bar{a}_{n}\right)+N_{c}\left(A_{1}, \bar{a}_{1}\right) \times \cdots \times N_{c}\left(A_{n}, \bar{a}_{n}\right)+\frac{\varepsilon^{\prime}}{\lambda} B_{\left(X^{n}\right)^{*}}
$$

where $g\left(x_{1}, \ldots, x_{n}\right):=\left(\sum_{i=1}^{n-1}\left\|x_{i}-x_{n}\right\|^{p}\right)^{\frac{1}{p}}$ for all $\left(x_{1}, \ldots, x_{n}\right) \in X^{n}$. Hence there exists $\left(-a_{1}^{*}, \ldots,-a_{n}^{*}\right) \in \partial g\left(\bar{a}_{1}, \ldots, \bar{a}_{n}\right)$ such that

$$
\left(\sum_{i=1}^{n} d\left(a_{i}^{*}, N_{c}\left(A_{i}, \bar{a}_{i}\right)\right)^{q}\right)^{\frac{1}{q}} \leq \frac{\varepsilon^{\prime}}{\lambda}<\frac{\varepsilon}{\lambda} .
$$

Thus,

$$
\sum_{i=1}^{n}\left\langle-a_{i}^{*}, x_{i}-\bar{a}_{i}\right\rangle \leq\left(\sum_{i=1}^{n-1}\left\|x_{i}-x_{n}\right\|^{p}\right)^{\frac{1}{p}}-\left(\sum_{i=1}^{n-1}\left\|\bar{a}_{i}-\bar{a}_{n}\right\|^{p}\right)^{\frac{1}{p}}
$$

for all $\left(x_{1}, \ldots, x_{n}\right) \in X^{n}$. Setting $x_{1}=\cdots=x_{n}=x$, it follows that

Copyright $($ ) by SIAM. Unauthorized reproduction of this article is prohibited. 


$$
\sum_{i=1}^{n}\left\langle-a_{i}^{*}, x-\bar{a}_{i}\right\rangle \leq-\left(\sum_{i=1}^{n-1}\left\|\bar{a}_{i}-\bar{a}_{n}\right\|^{p}\right)^{\frac{1}{p}} \quad \forall x \in X,
$$

and so $\sum_{i=1}^{n} a_{i}^{*}=0$. This and (3.4) imply that

$$
\sum_{i=1}^{n-1}\left\langle-a_{i}^{*}, x_{i}-x_{n}-\left(\bar{a}_{i}-\bar{a}_{n}\right)\right\rangle \leq\left(\sum_{i=1}^{n-1}\left\|x_{i}-x_{n}\right\|^{p}\right)^{\frac{1}{p}}-\left(\sum_{i=1}^{n-1}\left\|\bar{a}_{i}-\bar{a}_{n}\right\|^{p}\right)^{\frac{1}{p}}
$$

for all $\left(x_{1}, \ldots, x_{n}\right) \in X^{n}$. Taking an arbitrary element $\left(u_{1}, \ldots, u_{n-1}\right)$ in $X^{n-1}$ and letting $x_{i}:=u_{i}+x_{n}(1 \leq i \leq n-1)$, it follows that

$$
\sum_{i=1}^{n-1}\left\langle-a_{i}^{*}, u_{i}-\left(\bar{a}_{i}-\bar{a}_{n}\right)\right\rangle \leq\left(\sum_{i=1}^{n-1}\left\|u_{i}\right\|^{p}\right)^{\frac{1}{p}}-\left(\sum_{i=1}^{n-1}\left\|\bar{a}_{i}-\bar{a}_{n}\right\|^{p}\right)^{\frac{1}{p}}
$$

and so

$$
\left(-a_{1}^{*}, \ldots,-a_{n-1}^{*}\right) \in \partial\|\cdot\|_{X^{n-1}}\left(\bar{a}_{1}-\bar{a}_{n}, \ldots, \bar{a}_{n-1}-\bar{a}_{n}\right) .
$$

It follows from (3.3) that

$$
\left(\sum_{i=1}^{n-1}\left\|a_{i}^{*}\right\|^{q}\right)^{\frac{1}{q}}=1 \quad \text { and } \quad \sum_{i=1}^{n-1}\left\langle a_{i}^{*}, \bar{a}_{n}-\bar{a}_{i}\right\rangle=\left(\sum_{i=1}^{n-1}\left\|\bar{a}_{i}-\bar{a}_{n}\right\|^{p}\right)^{\frac{1}{p}} .
$$

This completes the proof. $\quad \square$

Theorem 3.1' Let $A_{1}, \ldots, A_{n}$, and $p, q$ be as in Theorem 3.1. Suppose that

$$
\left(\sum_{i=1}^{n-1}\left\|a_{i}-a_{n}\right\|^{p}\right)^{\frac{1}{p}}=\gamma_{p}\left(A_{1}, \ldots, A_{n}\right) .
$$

Then, there exist $a_{i}^{*} \in X^{*}(1 \leq i \leq n)$ with the following properties:

(i) $\left(\sum_{i=1}^{n-1}\left\|a_{i}^{*}\right\|^{q}\right)^{\frac{1}{q}}=1, \sum_{i=1}^{n} a_{i}^{*}=0$, and $a_{i}^{*} \in N_{c}\left(A_{i}, a_{i}\right), i=1, \ldots, n$.

(ii) $\left(\sum_{i=1}^{n-1}\left\|a_{n}-a_{i}\right\|^{p}\right)^{\frac{1}{p}}=\sum_{i=1}^{n-1}\left\langle a_{i}^{*}, a_{n}-a_{i}\right\rangle$.

Proof. Let $\phi$ and $g$ be as in the proof of Theorem 3.1. Then

$$
\phi\left(a_{1}, \ldots, a_{n}\right)=\inf \left\{\phi\left(x_{1}, \ldots, x_{n}\right):\left(x_{1}, \ldots, x_{n}\right) \in X^{n}\right\}
$$

Hence

$$
0 \in \partial \phi\left(a_{1}, \ldots, a_{n}\right) \subset \partial g\left(a_{1}, \ldots, a_{n}\right)+N_{c}\left(A_{1}, a_{1}\right) \times \cdots \times N_{c}\left(A_{n}, a_{n}\right) .
$$

It follows that $\left(a_{1}^{*}, \ldots, a_{n}^{*}\right) \in \partial g\left(x_{1}, \ldots, x_{n}\right)$ such that $a_{i}^{*} \in N_{c}\left(A_{i}, a_{i}\right)(i=1, \ldots, m)$. Noting that $\left(a_{1}-a_{n}, \ldots, a_{n-1}-a_{n}\right) \neq(0, \ldots, 0)$, as in the corresponding part of the proof of Theorem 3.1, one has

$$
\left(\sum_{i=1}^{n-1}\left\|a_{i}^{*}\right\|^{q}\right)^{\frac{1}{q}}=1 \quad \text { and } \quad \sum_{i=1}^{n-1}\left\langle a_{i}^{*}, a_{i}-a_{n}\right\rangle=\left(\sum_{i=1}^{n-1}\left\|a_{i}-a_{n}\right\|^{p}\right)^{\frac{1}{p}} .
$$

The proof is completed.

In view of Theorem 3.1, we have the following corollary.

Copyright (C) by SIAM. Unauthorized reproduction of this article is prohibited. 
Corollary 3.2. Let $A_{1}$ and $A_{2}$ be two closed nonempty subsets of $X$ such that $A_{1} \cap A_{2}=\varnothing$. Then, for any $\varepsilon>0$, there exist $a_{i} \in A_{i}(i=1,2)$ and $a^{*} \in X^{*}$ with $\left\|a^{*}\right\|=1$ such that

$$
-a^{*} \in N_{c}\left(A_{1}, a_{1}\right)+\varepsilon B_{X^{*}}, \quad a^{*} \in N_{c}\left(A_{2}, a_{2}\right)+\varepsilon B_{X^{*}}
$$

and

$$
\left\|a_{1}-a_{2}\right\|=\left\langle a^{*}, a_{1}-a_{2}\right\rangle<d\left(A_{1}, A_{2}\right)+\varepsilon .
$$

Remark. In Corollary $3.2, \varepsilon$ cannot be taken as 0 even in the convex setting. Indeed, there exist two closed convex sets $A_{1}$ and $A_{2}$ of $\mathbb{R}^{2}$ such that $d\left(A_{1}, A_{2}\right)>0$ but $N\left(A_{1}, a_{1}\right) \cap-N\left(A_{2}, a_{2}\right)=\{0\}$ for any $a_{1} \in A_{1}$ and $a_{2} \in A_{2}$. Let $A_{1}=\{(s, t) \in$ $\left.\mathbb{R}_{+}^{2} \backslash\{0\}: \frac{1}{s} \leq t\right\}$ and $A_{2}=\mathbb{R} \times[-1,-\infty)$. Then $A_{1}$ and $A_{2}$ are closed convex sets. It is clear that $\operatorname{bd}\left(A_{1}\right)=\left\{(s, t) \in \mathbb{R}_{+}^{2} \backslash\{0\}: t=\frac{1}{s}\right\}, \operatorname{bd}\left(A_{2}\right)=\mathbb{R} \times\{-1\}$, and $\mathbb{R} \times\{0\}$ is the asymptotic line of $\operatorname{bd}\left(A_{1}\right)$. Hence, $d\left(A_{1}, A_{2}\right)=1$,

$$
N\left(A_{1},(s, t)\right)=\mathbb{R}_{+}\left(-\frac{1}{s^{2}},-1\right) \quad \text { and } \quad N\left(A_{2},\left(s^{\prime}, t^{\prime}\right)\right)=\mathbb{R}_{+}(0,1)
$$

for all $(s, t) \in \operatorname{bd}\left(A_{1}\right)$ and all $\left(s^{\prime}, t^{\prime}\right) \in \operatorname{bd}\left(A_{2}\right)$. It follows that

$$
N\left(A_{1},(s, t)\right) \cap-N\left(A_{2},\left(s^{\prime}, t^{\prime}\right)\right)=\{(0,0)\} \quad \forall(s, t) \in A_{1} \quad \text { and } \quad \forall\left(s^{\prime}, t^{\prime}\right) \in A_{2} .
$$

Corollary 3.3. Let $A_{1}$ be a closed nonempty subset of $X$ and $A_{2}$ a closed, bounded, and convex nonempty subset of $X$. Suppose that $A_{1} \cap A_{2}=\varnothing$. Then, for any $\varepsilon>0$, there exist $a_{1} \in A_{1}$ and $a^{*} \in N_{c}\left(A_{1}, a_{1}\right)$ with $\left\|a^{*}\right\|=1$ such that

$$
d\left(A_{1}, A_{2}\right)-\varepsilon<\inf _{x \in A_{2}}\left\langle a^{*}, x\right\rangle-\left\langle a^{*}, a_{1}\right\rangle .
$$

Consequently, if in addition $A_{1}$ is convex, then

$$
d\left(A_{1}, A_{2}\right)-\varepsilon<\inf _{x \in A_{2}}\left\langle a^{*}, x\right\rangle-\max _{x \in A_{1}}\left\langle a^{*}, x\right\rangle .
$$

Proof. Let $k$ be an arbitrary natural number, and take $a_{i}(k) \in A_{i}$ such that

$$
\left\|a_{1}(k)-a_{2}(k)\right\|<d\left(A_{1}, A_{2}\right)+\frac{1}{k^{2}} ;
$$

that is,

$$
\left\|a_{1}(k)-a_{2}(k)\right\|<\gamma_{1}\left(A_{1}, A_{2}\right)+\frac{1}{k^{2}} .
$$

By Theorem 3.1, there exist $\bar{a}_{i}(k) \in A_{i}$ and $a_{i}^{*}(k) \in X^{*}$ such that

$$
\begin{gathered}
\left\|\bar{a}_{1}(k)-a_{1}(k)\right\|+\left\|\bar{a}_{2}(k)-a_{2}(k)\right\|<\frac{1}{k}, \\
\left\|a_{1}^{*}(k)\right\|=1, \quad a_{1}^{*}(k)+a_{2}^{*}(k)=0, \quad a_{i}^{*}(k) \in N_{c}\left(A_{i}, \bar{a}_{i}(k)\right)+\frac{1}{k} B_{X^{*}}, \quad i=1,2,
\end{gathered}
$$

Copyright $($ ) by SIAM. Unauthorized reproduction of this article is prohibited. 
and

$$
\left\|\bar{a}_{1}(k)-\bar{a}_{2}(k)\right\|=\left\langle a_{1}^{*}(k), \bar{a}_{2}(k)-\bar{a}_{1}(k)\right\rangle .
$$

Take $\bar{a}_{i}^{*}(k) \in N_{c}\left(A_{i}, \bar{a}_{i}(k)\right)$ such that $\left\|\bar{a}_{i}^{*}(k)-a_{i}^{*}(k)\right\|<\frac{1}{k}(i=1,2)$. Then

$$
1-\frac{1}{k}<\left\|\bar{a}_{i}^{*}(k)\right\|<1+\frac{1}{k}, \quad\left\|\bar{a}_{1}^{*}(k)+\bar{a}_{2}^{*}(k)\right\|<\frac{2}{k},
$$

and so

$$
\begin{aligned}
\left(1-\frac{1}{k}\right)\left\|\bar{a}_{1}(k)-\bar{a}_{2}(k)\right\| & \leq\left\langle\bar{a}_{1}^{*}(k), \bar{a}_{2}(k)-\bar{a}_{1}(k)\right\rangle \\
& \leq\left\langle-\bar{a}_{2}^{*}(k), \bar{a}_{2}(k)\right\rangle-\left\langle\bar{a}_{1}^{*}(k), \bar{a}_{1}(k)\right\rangle+\left\|\bar{a}_{1}^{*}(k)+\bar{a}_{2}^{*}(k)\right\|\left\|\bar{a}_{2}(k)\right\| \\
& \leq-\max _{x \in A_{2}}\left\langle\bar{a}_{2}^{*}(k), x\right\rangle-\left\langle\bar{a}_{1}^{*}(k), \bar{a}_{1}(k)\right\rangle+\frac{2 L}{k} \\
& \leq \inf _{x \in A_{2}}\left\langle\bar{a}_{1}^{*}(k), x\right\rangle-\left\langle\bar{a}_{1}^{*}(k), \bar{a}_{1}(k)\right\rangle+\frac{3 L}{k},
\end{aligned}
$$

where $L=\max _{x \in A_{2}}\|x\|$. Let $\tilde{a}^{*}(k):=\frac{\bar{a}_{1}^{*}(k)}{\left\|\bar{a}_{1}^{*}(k)\right\|}$. Then $\tilde{a}^{*}(k) \in N_{c}\left(A_{1}, \bar{a}_{1}(k)\right)$, and it follows that

$$
\frac{\left(1-\frac{1}{k}\right)\left\|\bar{a}_{1}(k)-\bar{a}_{2}(k)\right\|-\frac{3 L}{k}}{\left\|\bar{a}_{1}^{*}(k)\right\|} \leq \inf _{x \in A_{2}}\left\langle\tilde{a}^{*}(k), x\right\rangle-\left\langle\tilde{a}^{*}(k), \bar{a}_{1}(k)\right\rangle .
$$

By (3.5) and (3.6), one has

$$
\frac{\left(1-\frac{1}{k}\right)\left\|\bar{a}_{1}(k)-\bar{a}_{2}(k)\right\|-\frac{3 L}{k}}{\left\|\bar{a}_{1}^{*}(k)\right\|} \rightarrow d\left(A_{1}, A_{2}\right) .
$$

Hence

$$
d\left(A_{1}, A_{2}\right)-\varepsilon<\inf _{x \in A_{2}}\left\langle\tilde{a}^{*}(k), x\right\rangle-\left\langle\tilde{a}^{*}(k), \bar{a}_{1}(k)\right\rangle
$$

for all $k$ sufficiently large. The proof is completed.

Remark. In Corollary 3.3 , if $A_{2}$ is compact, then $d\left(A_{1}, A_{2}\right)>0$; taking $\varepsilon$ in $\left(0, d\left(A_{1}, A_{2}\right)\right)$, one can see that Corollary 3.3 improves and generalizes the convex separation theorem mentioned in section 1.

The following theorem implies that, when $X$ is an Asplund space, the Clarke normal cone in Theorem 3.1 can be replaced by the Fréchet normal cone, provided that the equality in Theorem 3.1(iii) is replaced with an inequality.

Theorem 3.4. Let $X$ be an Asplund space and $A_{1}, \ldots, A_{n}$ be closed nonempty subsets of $X$ such that $\bigcap_{i=1}^{n} A_{i}=\varnothing$. Let $1 \leq p, q \leq+\infty$ with $\frac{1}{p}+\frac{1}{q}=1, \varepsilon>0$, and $a_{i} \in A_{i}$ $(1 \leq i \leq n)$ be such that

$$
\left(\sum_{i=1}^{n-1}\left\|a_{i}-a_{n}\right\|^{p}\right)^{\frac{1}{p}}<\gamma_{p}\left(A_{1}, \ldots, A_{n}\right)+\varepsilon .
$$

Copyright ( by SIAM. Unauthorized reproduction of this article is prohibited. 
Then, for any $\lambda>0$ and any $\rho \in(0,1)$, there exist $\tilde{a}_{i} \in A_{i}$ and $a_{i}^{*} \in X^{*}$ with the following properties:

(i) $\left(\sum_{i=1}^{n}\left\|\tilde{a}_{i}-a_{i}\right\|^{p}\right)^{\frac{1}{p}}<\lambda$.

(ii) $\left(\sum_{i=1}^{n-1}\left\|a_{i}^{*}\right\|^{q}\right)^{\frac{1}{q}}=1, \sum_{i=1}^{n} a_{i}^{*}=0$, and $\left(\sum_{i=1}^{n} d\left(a_{i}^{*}, \hat{N}\left(A_{i}, \tilde{a}_{i}\right)\right)^{q}\right)^{\frac{1}{q}}<\frac{\varepsilon}{\lambda}$.

(iii) $\rho\left(\sum_{i=1}^{n-1}\left\|\tilde{a}_{i}-\tilde{a}_{n}\right\|^{p}\right)^{\frac{1}{p}} \leq \sum_{i=1}^{n-1}\left\langle a_{i}^{*}, \tilde{a}_{n}-\tilde{a}_{i}\right\rangle$.

Proof. Let $\phi$ be as in the proof of Theorem 3.1. Then

$$
\phi\left(a_{1}, \ldots, a_{n}\right)<\inf \left\{\phi\left(x_{1}, \ldots, x_{n}\right):\left(x_{1}, \ldots, x_{n}\right) \in X^{n}\right\}+\varepsilon .
$$

Take $\varepsilon^{\prime} \in(0, \varepsilon)$ and $\lambda^{\prime} \in(0, \lambda)$ such that

$$
\frac{\varepsilon^{\prime}}{\lambda^{\prime}}<\frac{\varepsilon}{\lambda} \quad \text { and } \quad \phi\left(a_{1}, \ldots, a_{n}\right)<\inf \left\{\phi\left(x_{1}, \ldots, x_{n}\right):\left(x_{1}, \ldots, x_{n}\right) \in X^{n}\right\}+\varepsilon^{\prime} .
$$

It follows from Ekeland's variational principle that there exists $\left(\bar{a}_{1}, \ldots, \bar{a}_{n}\right) \in X^{n}$ such that

$$
\left(\sum_{i=1}^{n}\left\|\bar{a}_{i}-a_{i}\right\|^{p}\right)^{\frac{1}{p}}<\lambda^{\prime}
$$

and

$$
\phi\left(\bar{a}_{1}, \ldots, \bar{a}_{n}\right) \leq \phi\left(x_{1}, \ldots, x_{n}\right)+\frac{\varepsilon^{\prime}}{\lambda^{\prime}}\left(\sum_{i=1}^{n}\left\|x_{i}-\bar{a}_{i}\right\|^{p}\right)^{\frac{1}{p}} \forall\left(x_{1}, \ldots, x_{n}\right) \in X^{n}
$$

Since $\bigcap_{i=1}^{n} A_{i}=\varnothing,(3.8)$ implies that

$$
\sum_{i=1}^{n-1}\left\|\bar{a}_{i}-\bar{a}_{n}\right\|>0
$$

For each $\left(x_{1}, \ldots, x_{n}\right) \in X^{n}$, let

$$
f\left(x_{1}, \ldots, x_{n}\right)=\left(\sum_{i=1}^{n-1}\left\|x_{i}-x_{n}\right\|^{p}\right)^{\frac{1}{p}}+\frac{\varepsilon^{\prime}}{\lambda^{\prime}}\left(\sum_{i=1}^{n}\left\|x_{i}-\bar{a}_{i}\right\|^{p}\right)^{\frac{1}{p}} .
$$

By (3.8) and the definition of $\phi$, one has $0 \in \hat{\partial}\left(f+\delta_{A_{1} \times \cdots \times A_{n}}\right)\left(\bar{a}_{1}, \ldots, \bar{a}_{n}\right)$. Let $0<\beta<\min \left\{\frac{\varepsilon}{\lambda}-\frac{\varepsilon^{\prime}}{\lambda^{\prime}}, \lambda-\lambda^{\prime}\right\}$. Then, by the Asplund space version of Lemma 2.1 and (3.9), there exist $\bar{x}_{i} \in X$ and $\tilde{a}_{i} \in A_{i}$ such that

$$
\left(\sum_{i=1}^{n}\left\|\bar{x}_{i}-\bar{a}_{i}\right\|^{p}\right)^{\frac{1}{p}}<\beta, \quad\left(\sum_{i=1}^{n}\left\|\tilde{a}_{i}-\bar{a}_{i}\right\|^{p}\right)^{\frac{1}{p}}<\beta, \quad 0<\sum_{i=1}^{n-1}\left\|\bar{x}_{i}-\bar{x}_{n}\right\|,
$$

and

$$
0 \in \hat{\partial} f\left(\bar{x}_{1}, \ldots, \bar{x}_{n}\right)+\hat{N}\left(A_{1} \times \cdots \times A_{n},\left(\tilde{a}_{1}, \ldots, \tilde{a}_{n}\right)\right)+\beta B_{X^{*}}^{n} .
$$

It follows from (3.7) that (i) holds. Let $g$ be as in the proof of Theorem 3.1. Then, $f=g+\frac{\varepsilon^{\prime}}{\lambda^{\prime}}\|\cdot\|_{X^{n}}$. By the convexity of $f$ and $g$, one has

Copyright ( by SIAM. Unauthorized reproduction of this article is prohibited. 


$$
\hat{\partial} f\left(\bar{x}_{1}, \ldots, \bar{x}_{n}\right)=\partial g\left(\bar{x}_{1}, \ldots, \bar{x}_{n}\right)+\frac{\varepsilon^{\prime}}{\lambda^{\prime}} B_{\left(X^{n}\right)^{*}}
$$

It follows from (3.11) that

$$
0 \in \partial g\left(\bar{x}_{1}, \ldots, \bar{x}_{n}\right)+\hat{N}\left(A_{1}, \tilde{a}_{1}\right) \times \cdots \times \hat{N}\left(A_{n}, \tilde{a}_{n}\right)+\left(\beta+\frac{\varepsilon^{\prime}}{\lambda^{\prime}}\right) B_{\left(X^{n}\right)^{*}}
$$

Hence there exists $-\left(a_{1}^{*}, \ldots, a_{n}^{*}\right) \in \partial g\left(\bar{x}_{1}, \ldots, \bar{x}_{n}\right)$ such that

$$
\left(\sum_{i=1}^{n} d\left(a_{i}^{*}, \hat{N}\left(A_{i}, \tilde{a}_{i}\right)\right)^{q}\right)^{\frac{1}{q}} \leq \beta+\frac{\varepsilon^{\prime}}{\lambda^{\prime}}<\frac{\varepsilon}{\lambda} .
$$

Noting (by the third inequality of $(3.10))$ that $\left(\bar{x}_{1}-\bar{x}_{n}, \ldots, \bar{x}_{n-1}-\bar{x}_{n}\right) \neq(0, \ldots, 0)$, as in the corresponding part of the proof of Theorem 3.1, one has

$$
\left(\sum_{i=1}^{n-1}\left\|a_{i}^{*}\right\|^{q}\right)^{\frac{1}{q}}=1 \quad \text { and } \quad \sum_{i=1}^{n-1}\left\langle a_{i}^{*}, \bar{x}_{n}-\bar{x}_{i}\right\rangle=\left(\sum_{i=1}^{n-1}\left\|\bar{x}_{i}-\bar{x}_{n}\right\|^{p}\right)^{\frac{1}{p}} .
$$

It follows from (3.10) that

$$
\begin{aligned}
\sum_{i=1}^{n-1}\left\langle a_{i}^{*}, \tilde{a}_{n}-\tilde{a}_{i}\right\rangle & =\sum_{i=1}^{n-1}\left\langle a_{i}^{*}, \bar{x}_{n}-\bar{x}_{i}\right\rangle+\sum_{i=1}^{n-1}\left\langle a_{i}^{*}, \tilde{a}_{n}-\bar{x}_{n}-\left(\tilde{a}_{i}-\bar{x}_{i}\right)\right\rangle \\
& \geq\left(\sum_{i=1}^{n-1}\left\|\bar{x}_{i}-\bar{x}_{n}\right\|^{p}\right)^{\frac{1}{p}}-\left(\sum_{i=1}^{n-1}\left\|\tilde{a}_{n}-\bar{x}_{n}-\left(\tilde{a}_{i}-\bar{x}_{i}\right)\right\|^{p}\right)^{\frac{1}{p}} \\
& \geq\left(\sum_{i=1}^{n-1}\left\|\tilde{a}_{i}-\tilde{a}_{n}\right\|^{p}\right)^{\frac{1}{p}}-2\left(\sum_{i=1}^{n-1}\left\|\tilde{a}_{n}-\bar{x}_{n}-\left(\tilde{a}_{i}-\bar{x}_{i}\right)\right\|^{p}\right)^{\frac{1}{p}} \\
& \geq\left(\sum_{i=1}^{n-1}\left\|\tilde{a}_{i}-\tilde{a}_{n}\right\|^{p}\right)^{\frac{1}{p}}-2\left(\sum_{i=1}^{n-1}(4 \beta)^{p}\right)^{\frac{1}{p}} \\
& \geq\left(\sum_{i=1}^{n-1}\left\|\tilde{a}_{i}-\tilde{a}_{n}\right\|^{p}\right)^{\frac{1}{p}}-8(n-1) \beta .
\end{aligned}
$$

Note that $\beta$ is arbitrary in $\left(0, \min \left\{\frac{\varepsilon}{\lambda}-\frac{\varepsilon^{\prime}}{\lambda^{\prime}}, \lambda-\lambda^{\prime}\right\}\right)$ and that (3.10) and (3.9) imply that

$$
\lim _{\beta \rightarrow 0^{+}}\left(\left(\sum_{i=1}^{n-1}\left\|\tilde{a}_{i}-\tilde{a}_{n}\right\|^{p}\right)^{\frac{1}{p}}-8(n-1) \beta\right)=\left(\sum_{i=1}^{n-1}\left\|\bar{a}_{i}-\bar{a}_{n}\right\|^{p}\right)^{\frac{1}{p}}>0 .
$$

By $\rho \in(0,1)$, one has

$$
\begin{aligned}
\lim _{\beta \rightarrow 0^{+}} \rho\left(\sum_{i=1}^{n-1}\left\|\tilde{a}_{i}-\tilde{a}_{n}\right\|^{p}\right)^{\frac{1}{p}} & =\rho\left(\sum_{i=1}^{n-1}\left\|\bar{a}_{i}-\bar{a}_{n}\right\|^{p}\right)^{\frac{1}{p}} \\
& <\lim _{\beta \rightarrow 0^{+}}\left(\left(\sum_{i=1}^{n-1}\left\|\tilde{a}_{i}-\tilde{a}_{n}\right\|^{p}\right)^{\frac{1}{p}}-8(n-1) \beta\right) .
\end{aligned}
$$

Copyright (C) by SIAM. Unauthorized reproduction of this article is prohibited. 
It follows that there exists $\beta \in\left(0, \min \left\{\frac{\varepsilon}{\lambda}-\frac{\varepsilon^{\prime}}{\lambda^{\prime}}, \lambda-\lambda^{\prime}\right\}\right)$ sufficiently small such that

$$
\rho\left(\sum_{i=1}^{n-1}\left\|\tilde{a}_{i}-\tilde{a}_{n}\right\|^{p}\right)^{\frac{1}{p}}<\left(\sum_{i=1}^{n-1}\left\|\tilde{a}_{i}-\tilde{a}_{n}\right\|^{p}\right)^{\frac{1}{p}}-8(n-1) \beta .
$$

Consequently $\rho\left(\sum_{i=1}^{n-1}\left\|\tilde{a}_{i}-\tilde{a}_{n}\right\|^{p}\right)^{\frac{1}{p}}<\sum_{i=1}^{n-1}\left\langle a_{i}^{*}, \tilde{a}_{i}-\tilde{a}_{n}\right\rangle$. The proof is completed.

Remark. The extremal principle by Mordukhovich et al. plays a key role in variational analysis in infinite dimensional spaces and deals with finitely many closed sets with a special common point (named as an extremal point). In contrast, Theorem A mentioned in section 1 deals with finitely many closed sets whose intersections are empty. As observed in [29, p. 1161, Remark], Theorem A improves the extremal principle. But, none of these fuzzy separation results can recapture the classical convex separation theorem even in the special case of a singleton and a closed convex set. We emphasize that the points $\tilde{a}_{i}$ and $a_{i}^{*}(i=1,2, \ldots, n)$ in Theorem 3.1 (and Theorem 3.4) satisfy properties (i), (ii), and (iii) simultaneously: if we only require these points to satisfy (i) and (ii), then the task is relatively easier and the contents of these theorems are basically the same as Theorem A, but to require the points to have the additional property (iii) makes the result even more interesting, so that it not only covers the existing fuzzy separation results but also recaptures the classical convex separation theorem mentioned in section 1. But, the existing fuzzy separation results cannot cover the convex separation theorem even in the special case of a singleton and a closed convex set.

In view of the above fuzzy separation theorem, we can establish approximate projection results as follows. In the special case when $n=1$, these approximate projection results have been known and played an important role in the study of error bound, metric regularity, and metric linear regularity for generalized equations (cf. [30], [31]).

Corollary 3.5. Let $X$ be an Asplund space and $A_{1}, \ldots, A_{n}$ be closed nonempty subsets of $X$. Let $x \in X \backslash \bigcap_{i=1}^{n} A_{i}$ and $\rho \in(0,1)$. Then there exist $a_{i} \in A_{i}$ and $a_{i}^{*} \in X^{*}(1 \leq i \leq n)$ such that the following assertions hold:

(i) $\max _{1 \leq i \leq n}\left\|a_{i}^{*}\right\|=1$ and $a_{i}^{*} \in \hat{N}\left(A_{i}, a_{i}\right)(1 \leq i \leq n)$.

(ii) $\rho \sum_{i=1}^{n}\left\|x-a_{i}\right\| \leq \min \left\{\sum_{i=1}^{n} d\left(x, A_{i}\right), \sum_{i=1}^{n}\left\langle a_{i}^{*}, x-a_{i}\right\rangle\right\}$.

Proof. For each natural number $k$, take $a_{i}(k) \in A_{i}(1 \leq i \leq n)$ such that

$$
\sum_{i=1}^{n}\left\|a_{i}(k)-x\right\|<\sum_{i=1}^{n} d\left(x, A_{i}\right)+\frac{1}{k^{2}}
$$

Let $A_{n+1}:=\{x\}$. Then $\gamma_{1}\left(A_{1}, \ldots, A_{n}, A_{n+1}\right)=\sum_{i=1}^{n} d\left(x, A_{i}\right)$ and

$$
\sum_{i=1}^{n}\left\|a_{i}(k)-x\right\|<\gamma_{1}\left(A_{1}, \ldots, A_{n}, A_{n+1}\right)+\frac{1}{k^{2}} .
$$

By Theorem 3.4 (applied to $a_{1}=a_{1}(k), \ldots, a_{n}=a_{n}(k), a_{n+1}=x, \varepsilon=\frac{1}{k^{2}}, \lambda=\frac{1}{k}$, and $\left.\rho=1-\frac{1}{k}\right)$, there exist $\tilde{a}_{i}(k) \in A_{i}$ and $a_{i}^{*}(k) \in X^{*}$ such that

$$
\begin{gathered}
\sum_{i=1}^{n}\left\|\tilde{a}_{i}(k)-a_{i}(k)\right\|<\frac{1}{k}, \\
\max _{1 \leq i \leq n}\left\|a_{i}^{*}(k)\right\|=1, \quad a_{i}^{*}(k) \in \hat{N}\left(A_{i}, \tilde{a}_{i}(k)\right)+\frac{1}{k} B_{X^{*}} \quad(1 \leq i \leq n),
\end{gathered}
$$

Copyright ( $\odot$ by SIAM. Unauthorized reproduction of this article is prohibited. 
and

$$
\left(1-\frac{1}{k}\right) \sum_{i=1}^{n}\left\|\tilde{a}_{i}(k)-x\right\| \leq \sum_{i=1}^{n}\left\langle a_{i}^{*}(k), x-\tilde{a}_{i}(k)\right\rangle .
$$

For each $i$, take $\tilde{a}_{i}^{*}(k) \in \hat{N}\left(A_{i}, \tilde{a}_{i}(k)\right)$ such that $\left\|\tilde{a}_{i}^{*}(k)-a_{i}^{*}(k)\right\| \leq \frac{1}{k}$, and let $\eta_{k}:=$ $\max _{1 \leq i \leq n}\left\|\tilde{a}_{i}^{*}(k)\right\|$. It follows from (3.15) that

$$
\frac{1}{\eta_{k}}\left(1-\frac{2}{k}\right) \sum_{i=1}^{n}\left\|\tilde{a}_{i}(k)-x\right\| \leq \sum_{i=1}^{n}\left\langle\frac{\tilde{a}_{i}^{*}(k)}{\eta_{k}}, x-\tilde{a}_{i}(k)\right\rangle .
$$

Clearly, (3.12) and (3.13) imply that

$$
\sum_{i=1}^{n}\left\|\tilde{a}_{i}(k)-x\right\|<\sum_{i=1}^{n} d\left(x, A_{i}\right)+\frac{1}{k}+\frac{1}{k^{2}} .
$$

Noting that $\eta_{k} \rightarrow 1$ as $k \rightarrow \infty, \rho \in(0,1)$, and $0<\sum_{i=1}^{n} d\left(x, A_{i}\right) \leq \sum_{i=1}^{n}\left\|\tilde{a}_{i}(k)-x\right\|$ for all $k$, it follows from (3.16) that

$$
\rho \sum_{i=1}^{n}\left\|\tilde{a}_{i}(k)-x\right\| \leq \min \left\{\sum_{i=1}^{n} d\left(x, A_{i}\right), \sum_{i=1}^{n}\left\langle\frac{\tilde{a}_{i}^{*}(k)}{\eta_{k}}, x-\tilde{a}_{i}(k)\right\rangle\right\}
$$

for all $k$ sufficiently large. The proof is completed.

Similar to the proof of Corollary 3.5 (with Theorem 3.1 replacing Theorem 3.4), one can prove the following result.

Corollary 3.6. Let $X$ be a general Banach space and $A_{1}, \ldots, A_{n}$ be closed nonempty subsets of $X$. Let $x \in X \backslash \bigcap_{i=1}^{n} A_{i}$ and $\rho \in(0,1)$. Then there exist $a_{i} \in A_{i}$ and $a_{i}^{*} \in X^{*}(1 \leq i \leq n)$ such that the following assertions hold:

(i) $\max _{1 \leq i \leq n}\left\|a_{i}^{*}\right\|=1$ and $a_{i}^{*} \in N_{c}\left(A_{i}, a_{i}\right)(1 \leq i \leq n)$.

(ii) $\rho \sum_{i=1}^{n}\left\|a_{i}-x\right\| \leq \min \left\{\sum_{i=1}^{n} d\left(x, A_{i}\right), \sum_{i=1}^{n}\left\langle a_{i}^{*}, a_{i}-x\right\rangle\right\}$.

Theorems 3.1 and 3.4 unify the classical convex separation theorem and existing fuzzy separation results mentioned in section 1 .

4. Application to multiobjective optimization. Let $Y$ be a Banach space and $K$ be a closed convex pointed cone in $Y$, which specifies a partial order $\leq_{K}$ on $Y$ as follows: for $y_{1}, y_{2} \in Y$,

$$
y_{1} \leq_{K} y_{2} \quad \text { if and only if } \quad y_{2}-y_{1} \in K .
$$

Let $K^{+}$denote the dual cone of $K$; that is,

$$
K^{+}:=\left\{y^{*} \in Y^{*}: 0 \leq\left\langle y^{*}, y\right\rangle \forall y \in K\right\} .
$$

Let $Z$ be a subset of $Y$ and recall that $y \in Z$ is said to be a Pareto efficient point, written as $y \in \mathrm{E}(Z, K)$, if $z \in Z$ and $z \leq_{K} y \Rightarrow z=y$. It is known and easy to verify that

$$
y \in E(Z, K) \Leftrightarrow(Z+K) \cap(y-K)=\{y\} .
$$

In the case when $\operatorname{int}(K) \neq \varnothing$, recall that $y \in Z$ is said to be a weak Pareto efficient point, written as $y \in \mathrm{WE}(Z, K)$, if

Copyright ( by SIAM. Unauthorized reproduction of this article is prohibited. 


$$
(Z+K) \cap(y-\operatorname{int}(K))=\varnothing .
$$

Throughout this section, let $X, Y_{0}, Y_{1}, \ldots, Y_{m}$ be Banach spaces, $\Phi_{i}: X \rightrightarrows Y_{i}$ $(i=0,1, \ldots, m)$ be closed multifunctions, $A$ be a closed subset of $X$, and $K_{i}$ be a closed convex cone in $Y_{i}(i=0,1, \ldots, m)$. We consider the following constraint vector optimization problem:

$$
\begin{aligned}
& K_{0}-\min \Phi_{0}(x), \\
& \Phi_{i}(x) \cap-K_{i} \neq \varnothing, \quad i=1, \ldots, m, \\
& x \in A .
\end{aligned}
$$

In the special case when $Y_{0}=\cdots=Y_{m}=\mathbb{R}, \quad K_{0}=\cdots=K_{n}=\mathbb{R}_{+}, \quad K_{n+1}=\cdots=$ $K_{m}=\{0\}$, and each $\Phi_{i}$ is single-valued, (4.1) reduces to the usual constraint numerical optimization problem. In the remainder of this section, suppose that $K_{0}$ is pointed, and let $Z$ denote the feasible set of (4.1); that is,

$$
Z:=A \cap\left(\bigcap_{i=}^{m} \Phi_{i}^{-1}\left(-K_{i}\right)\right) .
$$

For $\bar{x} \in Z$ and $\bar{y} \in \Phi_{0}(\bar{x})$, we say that $(\bar{x}, \bar{y})$ is a Pareto solution (resp., weak Pareto solution) of vector optimization problem (4.1) if

$$
\bar{y} \in \mathrm{E}\left(\Phi_{0}(Z), K_{0}\right) \quad\left(\text { resp., } \bar{y} \in \mathrm{WE}\left(\Phi_{0}(Z), K_{0}\right)\right)
$$

that is,

$$
\begin{gathered}
\Phi_{0}(Z) \cap\left(\bar{y}-K_{0}\right)=\{\bar{y}\} \\
\left(\text { resp., } \Phi_{0}(Z) \cap\left(\bar{y}-\operatorname{int}\left(K_{0}\right)\right)=\varnothing\right) .
\end{gathered}
$$

It is well known that

$$
\bar{y} \in \mathrm{E}\left(\Phi_{0}(Z), K_{0}\right) \Leftrightarrow\left(\Phi_{0}(Z)+K_{0}\right) \cap\left(\bar{y}-K_{0}\right)=\{\bar{y}\} .
$$

Many authors have established sufficient or necessary optimality conditions for Pareto solutions and weak Pareto solutions of constraint vector optimization (4.1) (see [3], [5], [6], [17], [21], [26], [27], [28], [29], [32], and references therein). In general, even in the case when $Y_{0}=\cdots=Y_{n}, K_{0}=\cdots=K_{n}=\mathbb{R}_{+}$, and each $\Phi_{i}$ is single-valued, (4.1) need not have a (Pareto or weak Pareto) solution if $X$ is infinite dimensional. So it is natural and interesting to consider some kinds of approximate solutions. Let $\Phi_{0}: X \rightarrow \mathbb{R} \cup\{+\infty\}$ be a proper lower semicontinuous function bounded below on $Z$. For $\varepsilon>0$, one naturally defines that $\bar{x} \in Z$ is an $\varepsilon$-approximate solution of the following problem:

$$
\min _{x \in Z} \Phi_{0}(x)
$$

if

$$
\Phi_{0}(\bar{x})<\inf \left\{\Phi_{0}(x): x \in Z\right\}+\varepsilon,
$$

or equivalently,

Copyright ( $)$ by SIAM. Unauthorized reproduction of this article is prohibited. 


$$
\operatorname{diam}\left(\left(\Phi_{0}(Z)+\mathbb{R}_{+}\right) \cap\left(\Phi_{0}(a)-\mathbb{R}_{+}\right)\right)<\varepsilon
$$

Motivated by (4.3) and (4.4), we introduce the following notions of an approximate Pareto solution for vector optimization problem (4.1).

Definition 4.1. Let $\varepsilon>0, \bar{x} \in Z$, and $\bar{y}_{0} \in \Phi_{0}(\bar{x})$. We say that $\left(\bar{x}, \bar{y}_{0}\right)$ is

(i) an $\varepsilon$-Pareto solution of (4.1) if

$$
\operatorname{diam}\left(\left(\Phi_{0}(Z)+K_{0}\right) \cap\left(\bar{y}_{0}-K_{0}\right)\right)<\varepsilon,
$$

(ii) a weak $\varepsilon$-Pareto solution of (4.1) if there exists $e \in \varepsilon B_{Y_{0}}$ such that

$$
\Phi_{0}(Z) \cap\left(\bar{y}_{0}+e-K_{0}\right)=\varnothing
$$

(whether $\operatorname{int}\left(K_{0}\right)$ is empty or not).

Proposition 4.1. Let $\varepsilon>0, \bar{x} \in Z$, and $\bar{y}_{0} \in \Phi_{0}(\bar{x})$ be such that $\left(\bar{x}, \bar{y}_{0}\right)$ is an $\varepsilon$-Pareto solution of (4.1). Then, (4.6) holds for any $e \in-K_{0}$ with $\|e\| \geq \varepsilon$. Consequently, $\left(\bar{x}, \bar{y}_{0}\right)$ is a weak $\varepsilon$-Pareto solution of (4.1).

Proof. Take an arbitrary $e$ in $-K_{0}$ with $\|e\| \geq \varepsilon$. Noting that $\bar{y}_{0} \in\left(\Phi_{0}(Z)+K_{0}\right) \cap$ $\left(\bar{y}_{0}-K_{0}\right)$ and $\left\|\bar{y}_{0}-\left(\bar{y}_{0}+e\right)\right\|=\|e\| \geq \varepsilon,(4.5)$ implies that $\bar{y}_{0}+e \notin\left(\Phi_{0}(Z)+K_{0}\right) \cap$ $\left(\bar{y}_{0}-K_{0}\right)$, and so

$$
\left(\Phi_{0}(Z)+K_{0}\right) \cap\left(\bar{y}_{0}+e-K_{0}\right)=\varnothing .
$$

This shows that $\left(\bar{x}, \bar{y}_{0}\right)$ is a weak $\varepsilon$-Pareto solution of (4.1).

By Definition 4.1, it is clear that if $\left(\bar{x}, \bar{y}_{0}\right)$ is a Pareto solution of $(4.1)$, then $\left(\bar{x}, \bar{y}_{0}\right)$ is an $\varepsilon$-Pareto solution of (4.1) for any $\varepsilon>0$. In the case when $\operatorname{int}\left(K_{0}\right) \neq \varnothing$, noting that $e-K_{0} \subset-\operatorname{int}\left(K_{0}\right)$ for each $e \in-\operatorname{int}\left(K_{0}\right)$, it is easy from Definition 4.1 to verify that if $\left(\bar{x}, \bar{y}_{0}\right)$ is a weak Pareto solution of $(4.1)$, then $\left(\bar{x}, \bar{y}_{0}\right)$ is a weak $\varepsilon$-Pareto solution of $(4.1)$ for any $\varepsilon>0$. The following example shows that $\left(\bar{x}, \bar{y}_{0}\right)$ is not necessarily an $\varepsilon$-Pareto solution of (4.1) when $\left(\bar{x}, \bar{y}_{0}\right)$ is a weak Pareto solution of (4.1).

Example. Let $X=Y_{0}=\cdots=Y_{m}=\mathbb{R}^{2}, K_{1}=\cdots=K_{m}=\mathbb{R}^{2}, K_{0}=\mathbb{R}_{+}^{2}, A=\mathbb{R}^{2}$, $\Phi_{1}=\cdots=\Phi_{m}=I_{\mathbb{R}^{2}}$, and

$$
\Phi_{0}(s, t)=\{(s, 0)\} \quad \forall(s, t) \in \mathbb{R}^{2} .
$$

Then, $Z=\mathbb{R}^{2}$ and $\Phi_{0}(Z)+K_{0}=\mathbb{R} \times \mathbb{R}_{+}$. Let $(s, t) \in Z$. It is easy to verify that $((s, t),(s, 0))$ is a weak Pareto solution of $(4.1)$ and

$$
\left(\Phi_{0}(Z)+K_{0}\right) \cap\left((s, 0)-K_{0}\right)=(-\infty, s] \times\{0\},
$$

and so $\operatorname{diam}\left(\left(\Phi_{0}(Z)+K_{0}\right) \cap\left((s, 0)-K_{0}\right)\right)=+\infty$. Hence $((s, t),(s, 0))$ is not an $\varepsilon$ Pareto solution of (4.1) for any $\varepsilon>0$.

Given a fixed $e_{0} \in K_{0} \backslash\{0\}$, in 1979, Kutateladze introduced the concept of an $\left(\varepsilon, e_{0}\right)$-minimizer of $\Phi_{0}(Z)$ with respect to $K_{0}: \bar{y}_{0} \in \Phi_{0}(Z)$ is said to be an $\left(\varepsilon, e_{0}\right)$-minimizer of $\Phi_{0}(Z)$ if (4.6) holds for $e=-\varepsilon e_{0}$. Kutateladze's concept is a very popular kind of $\varepsilon$-solution in vector optimization (see [1], [2] for the details). Several authors considered other kinds of $\varepsilon$-solutions for vector optimization (see [9], [19], [24], [25]). Recently, Gutierrez, Jiménez, and Novo [8] introduced a new $\varepsilon$-solution concept that extends many $\varepsilon$-solution notions introduced in the literature. Most of the existing approximate solutions are weaker than the $\varepsilon$-Pareto solution and stronger than the weak $\varepsilon$-Pareto solution. We will provide some necessary conditions for the existence of weak $\varepsilon$-Pareto solutions and some sufficient conditions for the existence of $\varepsilon$-Pareto solutions.

Copyright (c) by SIAM. Unauthorized reproduction of this article is prohibited. 
It is trivial that if a real-valued function $\phi: X \rightarrow \mathbb{R}$ is bounded below over a subset $Z$ of $X$, then for any $\varepsilon>0$ there exists $x_{\varepsilon} \in Z$ such that

$$
\phi\left(x_{\varepsilon}\right)-\varepsilon<\phi(x) \quad \forall x \in Z .
$$

It is natural to ask whether the corresponding result on $\varepsilon$-Pareto solutions for vector optimization is true. The following example says that the answer to this problem is negative.

Example. Let $X=\mathbb{R}, p \in[1,+\infty), Y=l^{p}$, and $F: X \rightrightarrows Y$ be such that

$$
F(x)=\left\{\left(\frac{1}{|x|+1}, 0,0, \ldots\right)\right\} \forall x \in X
$$

Then $F$ is a continuous single-valued function. Let $Z=X$ and $K_{0}$ consist of all $y=\left(t_{1}, t_{2}, \ldots\right) \in l^{p}$ such that $\sum_{k=1}^{n} t_{k} \geq 0$ for each $n \in \mathbb{N}$. It is clear that $K_{0}$ is a closed convex pointed cone in $Y$, and 0 is a lower bound of $F$ over $Z$ with respect to $K_{0}$. Now we show that (4.1) has no $\varepsilon$-Pareto solution for any $\varepsilon>0$. Indeed, let $\bar{x} \in X, \bar{u}=2|\bar{x}|+1$, $\bar{y}:=\left(\frac{1}{|\bar{x}|+1}, 0, \ldots\right)$, and $\bar{z}:=\left(\frac{1}{2(|\bar{x}|+1)}, 0, \ldots\right)$. Then $F(\bar{x})=\{\bar{y}\}$ and $F(\bar{u})=\{\bar{z}\}$. For any $n \in \mathbb{N}$, let $y_{n}:=\left(0, s_{1}, \ldots, s_{2 n}, 0, \ldots\right)$ be such that $s_{2 k-1}=-\frac{1}{4(|\bar{x}|+1)}$ and $s_{2 k}=\frac{1}{4(|\bar{x}|+1)} \times(k=1, \ldots, n)$. It is easy to verify that

$$
\bar{y}+y_{n} \in\left(\bar{z}+K_{0}\right) \cap\left(\bar{y}-K_{0}\right) \subset\left(F(Z)+K_{0}\right) \cap\left(\bar{y}-K_{0}\right) \quad \forall n \in \mathbb{N} .
$$

Noting that $\bar{y} \in\left(F(Z)+K_{0}\right) \cap\left(\bar{y}-K_{0}\right)$, it follows that

$$
\operatorname{diam}\left(\left(F(Z)+K_{0}\right) \cap\left(\bar{y}-K_{0}\right)\right) \geq\left\|y_{n}\right\|=\frac{n}{2(|\bar{x}|+1)} \rightarrow+\infty .
$$

This shows that $(\bar{x}, \bar{y})$ is not an $\varepsilon$-Pareto solution of (4.1) for any $\varepsilon>0$.

We will show that (4.1) always has a weak $\varepsilon$-Pareto solution if the objective multifunction $\Phi_{0}$ is bounded below on the feasible set $Z$ with respect to $K_{0}$. Moreover, under the mild assumption on the ordering cone, we can establish the same result for $\varepsilon$-Pareto solutions. To do this, recall that a closed convex cone $K$ of a Banach space $Y$ is said to have a bounded base if there exists a bounded closed convex subset $\Theta$ of $K$ such that

$$
0 \notin \Theta \quad \text { and } \quad K=\{t \theta: t \geq 0 \text { and } \theta \in \Theta\} .
$$

It is known that every closed convex pointed cone in a finite dimensional Banach space has a bounded base (cf. [11]).

Proposition 4.2. Let the objective multifunction $\Phi_{0}$ be bounded below on the feasible set $Z$ with respect to $K_{0}$; that is, there exists $b \in Y_{0}$ such that

$$
b \leq_{K_{0}} y \quad \forall y \in \Phi_{0}(Z) .
$$

Then the following statements hold:

(i) For any $\varepsilon>0,(4.1)$ always has a weak $\varepsilon$-Pareto solution.

(ii) If, in addition, $K_{0}$ has a bounded base, then, for any $\varepsilon>0$, (4.1) has an $\varepsilon$-Pareto solution.

Proof. Note that $K_{0}$ is pointed and $K_{0} \neq\{0\}$. Hence there exist $y_{0}^{*} \in K_{0}^{+}$and $c_{0} \in$ $K_{0}$ such that $\left\langle y_{0}^{*}, c_{0}\right\rangle>0$. Since $\Phi_{0}$ is bounded below on $Z$ with respect to $K_{0}$, for any $\varepsilon>0$, there exist $\bar{x} \in Z$ and $\bar{y}_{0} \in \Phi_{0}(\bar{x})$ such that

Copyright ( by SIAM. Unauthorized reproduction of this article is prohibited. 


$$
\left\langle y_{0}^{*}, \bar{y}_{0}\right\rangle<\inf _{y \in \Phi_{0}(Z)}\left\langle y_{0}^{*}, y\right\rangle+\left\langle y_{0}^{*}, \frac{\varepsilon c_{0}}{\left\|c_{0}\right\|}\right\rangle .
$$

It follows that $\Phi_{0}(Z) \cap\left(\bar{y}_{0}-\frac{\varepsilon c_{0}}{\left\|c_{0}\right\|}-K_{0}\right)=\varnothing$. Hence $\left(\bar{x}, \bar{y}_{0}\right)$ is a weak $\varepsilon$-Pareto solution of (4.1). This shows that (i) holds.

To prove (ii), suppose that $K_{0}$ has a bounded base. Hence there exists a bounded closed convex subset $\Theta$ of $K_{0}$ such that (4.7) holds. By the separation theorem, there exists $y_{0}^{*} \in Y_{0}^{*}$ with $\left\|y_{0}^{*}\right\|=1$ such that

$$
\eta:=\inf _{\theta \in \Theta}\left\langle y_{0}^{*}, \theta\right\rangle>0 .
$$

This and (4.7) imply that $y_{0}^{*} \in K_{0}^{+}$. Since $\Phi_{0}$ is bounded below on $Z$ with respect to $K_{0}$, $y_{0}^{*}$ is bounded below on $\Phi_{0}(Z)$ and hence is bounded below on $\Phi_{0}(Z)+K_{0}$. Let $\varepsilon$ be an arbitrary positive number and take $\bar{x} \in Z$ and $\bar{y}_{0} \in \Phi_{0}(Z)$ such that

$$
\left\langle y_{0}^{*}, \bar{y}_{0}\right\rangle-\frac{\eta \varepsilon}{2 M}<\inf _{y \in \Phi_{0}(Z)+K_{0}}\left\langle y_{0}^{*}, y\right\rangle
$$

where $M=\sup _{\theta \in \Theta}\|\theta\|$. On the other hand, (4.8) implies that

$$
\left\langle y_{0}^{*}, \bar{y}_{0}-t \theta\right\rangle \leq\left\langle y_{0}^{*}, \bar{y}_{0}\right\rangle-\frac{\varepsilon \eta}{2 M} \quad \forall(t, \theta) \in\left[\frac{\varepsilon}{2 M},+\infty\right) \times \Theta .
$$

It follows from (4.9) that $\bar{y}_{0}-t \theta \notin \Phi_{0}(Z)+K_{0}$ for any $(t, \theta) \in\left[\frac{\varepsilon}{2 M},+\infty\right) \times \Theta$; that is,

$$
\left(\Phi_{0}(Z)+K_{0}\right) \cap\left(\bar{y}_{0}-K_{0}\right) \subset\left(0, \frac{\varepsilon}{2 M}\right) \Theta .
$$

Hence $\operatorname{diam}\left(\left(\Phi_{0}(Z)+K_{0}\right) \cap\left(\bar{y}_{0}-K_{0}\right)\right) \leq \frac{\varepsilon}{2}$. This shows that $\left(\bar{x}, \bar{y}_{0}\right)$ is an $\varepsilon$-Pareto solution of (4.1). The proof is completed.

For various types of approximate solutions for (4.1), the following implications indicated in the diagram hold (" $=\Rightarrow$ " is under the assumption that $\left.\operatorname{int}\left(K_{0}\right) \neq \varnothing\right)$ :



Remark. All "reverve implications" are not valid, and "weak Pareto" does not imply " $\varepsilon$-Pareto."

Let $\phi: X \rightarrow \mathbb{R}$ be a real-valued Lipschtiz function such that $\phi$ is bounded below on $Z$. It is well known (cf. [4]) that for any $\varepsilon>0$ there exists $a_{\varepsilon} \in A$ such that

$$
\phi\left(a_{\varepsilon}\right)<\inf _{x \in Z} \phi(x)+\varepsilon \quad \text { and } \quad d\left(0, \partial_{c} \phi\left(a_{\varepsilon}\right)+N_{c}\left(Z, a_{\varepsilon}\right)\right)<\varepsilon .
$$

In this section, based on fuzzy separations obtained in section 3, we consider the corresponding issues for multiobjective optimization problem (4.1). To do this, we first provide the Lagrange-like multiplier rule for a weak $\varepsilon$-Pareto solution of (4.1).

Theorem 4.3. Let $\varepsilon>0$ and $\left(\bar{x}, \bar{y}_{0}\right)$ be a weak $\varepsilon$-Pareto solution of (4.1). Let $\bar{y}_{i} \in$ $\Phi_{i}(\bar{x}) \cap-K_{i}(i=1, \ldots, m)$. Then, for any $\lambda>0$ there exist $x_{i} \in B(\bar{x}, \lambda), y_{i} \in \Phi_{i}\left(x_{i}\right) \cap$ $B\left(\bar{y}_{i}, \lambda\right), x_{m+1} \in A \cap B(\bar{x}, \lambda), c_{i}^{*} \in K_{i}^{+}$,

Copyright ( ) by SIAM. Unauthorized reproduction of this article is prohibited. 


$$
\begin{aligned}
x_{i}^{*} & \in D_{c}^{*} \Phi_{i}\left(x_{i}, y_{i}\right)\left(c_{i}^{*}+\frac{\varepsilon}{\lambda} B_{Y_{i}^{*}}\right)+\frac{\varepsilon}{\lambda} B_{X^{*}}(0 \leq i \leq m), \quad \text { and } \\
x_{m+1}^{*} & \in N_{c}\left(A, x_{m+1}\right)+\frac{\varepsilon}{\lambda} B_{X^{*}}
\end{aligned}
$$

such that $\sum_{i=0}^{m+1} x_{i}^{*}=0$ and

$$
\frac{1}{2}-\frac{\varepsilon}{\lambda}<\sum_{i=0}^{m}\left(\left\|x_{i}^{*}\right\|+\left\|c_{i}^{*}\right\|\right)<1+\frac{\varepsilon}{\lambda}
$$

Proof. Since $\left(\bar{x}, \bar{y}_{0}\right)$ is a weak $\varepsilon$-Pareto solution of (4.1), there exists $e \in Y_{0}$ with $\|e\|<\varepsilon$ such that (4.6) holds. Equip the product space $X \times \prod_{i=0}^{m} Y_{i}$ with the following norm:

$$
\left\|\left(x, y_{0}, \ldots, y_{m}\right)\right\|:=\max \left\{\|x\|, \max _{0 \leq i \leq m}\left\|y_{i}\right\|\right\} \quad \forall\left(x, y_{0}, \ldots, y_{m}\right) \in X \times \prod_{i=0}^{m} Y_{i}
$$

and let

$$
\begin{gathered}
A_{i}:=\left\{\left(x, y_{0}, \ldots, y_{m}\right) \in X \times \prod_{i=0}^{m} Y_{i}:\left(x, y_{i}\right) \in \operatorname{Gr}\left(\Phi_{i}\right)\right\} \quad(i=0,1, \ldots, m), \\
A_{m+1}:=A \times\left(\bar{y}_{0}+e-K_{0}\right) \times \prod_{i=1}^{m}\left(\bar{y}_{i}-K_{i}\right) .
\end{gathered}
$$

We claim that $\bigcap_{i=0}^{m+1} A_{i}=\varnothing$. To do this, suppose to the contrary that there exist $\tilde{x} \in A$ and $\tilde{y}_{i} \in \Phi_{i}(\tilde{x})(i=0,1, \ldots, m)$ such that

$$
\tilde{y}_{0} \in \bar{y}_{0}+e-K_{0} \quad \text { and } \quad \tilde{y}_{i} \in \bar{y}_{i}-K_{i} \subset-K_{i} \quad(i=1, \ldots, m) .
$$

It follows that $\tilde{x} \in A \cap\left(\bigcap_{i=1}^{m} \Phi_{i}^{-1}\left(-K_{i}\right)\right)=Z$, and so

$$
\tilde{y}_{0} \in \Phi_{0}(Z) \cap\left(\bar{y}_{0}+e-K_{0}\right),
$$

contradicting (4.6). This shows that $\bigcap_{i=0}^{m+1} A_{i}=\varnothing$. Let

$$
a_{0}=\cdots=a_{m}=\left(\bar{x}, \bar{y}_{0}, \bar{y}_{1}, \ldots, \bar{y}_{m}\right) \quad \text { and } \quad a_{m+1}=\left(\bar{x}, \bar{y}_{0}+e, \bar{y}_{1}, \ldots, \bar{y}_{m}\right) \text {. }
$$

Then

$$
\max _{0 \leq i \leq m}\left\|a_{i}-a_{m+1}\right\|=\|e\|<\varepsilon \leq \gamma_{\infty}\left(A_{0}, A_{1}, \ldots, A_{m+1}\right)+\varepsilon .
$$

By Theorem 3.1, there exist $\bar{a}_{i}=\left(x_{i}, y_{i, 0}, \ldots, y_{i, m}\right) \in A_{i}$ and $\left(x_{i}^{*}, y_{i, 0}^{*}, \ldots, y_{i, m}^{*}\right) \in$ $\left(X \times Y_{0} \times \cdots \times Y_{m+1}\right)^{*}$ such that

$$
\sum_{i=0}^{m+1} d\left(\left(x_{i}^{*}, y_{i, 0}^{*}, \ldots, y_{i, m}^{*}\right), N_{c}\left(A_{i}, \bar{a}_{i}\right)\right)<\frac{\varepsilon}{\lambda},
$$

Copyright ( by SIAM. Unauthorized reproduction of this article is prohibited. 


$$
\sum_{i=0}^{m+1}\left(\left\|x_{i}^{*}\right\|+\sum_{k=0}^{m}\left\|y_{i, k}^{*}\right\|\right)=1
$$

and

$$
\sum_{i=0}^{m+1}\left(x_{i}^{*}, y_{i, 0}^{*}, \ldots, y_{i, m}^{*}\right)=0 .
$$

By the definition of each $A_{i}$, one has

$$
N_{c}\left(A_{m+1}, \bar{a}_{m+1}\right) \subset N_{c}\left(A, x_{m+1}\right) \times \prod_{i=0}^{m} K_{i}^{+}
$$

and

$$
N_{c}\left(A_{i}, \bar{a}_{i}\right)=\left\{\left(x^{*}, y_{0}^{*}, \ldots, y_{m}^{*}\right):\left(x^{*}, y_{i}^{*}\right) \in N_{c}\left(\operatorname{Gr}\left(\Phi_{i}\right),\left(x_{i}, y_{i, i}\right)\right) \text { and } y_{k}^{*}=0 \forall k \neq i\right\}
$$

for $0 \leq i \leq m$. This and (4.11) imply that there exist

$$
\begin{gathered}
\left(\tilde{x}_{i}^{*}, \tilde{y}_{i}^{*}\right) \in N_{c}\left(\operatorname{Gr}\left(\Phi_{i}\right),\left(x_{i}, y_{i, i}\right)\right) \quad(0 \leq i \leq m), \\
\tilde{x}_{m+1}^{*} \in N_{c}\left(A, x_{m+1}\right), \quad \text { and } \quad\left(c_{0}^{*}, \ldots, c_{m}^{*}\right) \in \prod_{k=0}^{m} K_{k}^{+}
\end{gathered}
$$

such that

$$
\sum_{i=0}^{m+1}\left\|\tilde{x}_{i}^{*}-x_{i}^{*}\right\|+\sum_{i=0}^{m}\left\|\tilde{y}_{i}^{*}-y_{i, i}^{*}\right\|+\sum_{i, k=0, k \neq i}^{m}\left\|y_{i, k}^{*}\right\|+\sum_{k=0}^{m}\left\|y_{m+1, k}^{*}-c_{k}^{*}\right\|<\frac{\varepsilon}{\lambda} .
$$

It follows from (4.14) that

$$
-\tilde{y}_{k}^{*}=c_{k}^{*}+\left(y_{k, k}^{*}-\tilde{y}_{k}^{*}\right)+\left(y_{m+1, k}^{*}-c_{k}^{*}\right)+\sum_{i=0, i \neq k}^{m} y_{i, k}^{*} \in c_{k}^{*}+\frac{\varepsilon}{\lambda} B_{Y_{k}^{*}}, \quad 0 \leq k \leq m .
$$

By (4.14)-(4.17), one has

$$
\begin{gathered}
x_{k}^{*} \in D_{c}^{*} \Phi_{k}\left(x_{k}, y_{k, k}\right)\left(c_{k}^{*}+\frac{\varepsilon}{\lambda} B_{Y_{k}^{*}}\right)+\frac{\varepsilon}{\lambda} B_{X^{*}}, \quad k=0,1 \ldots, m, \\
x_{m+1}^{*} \in N_{c}\left(A, x_{m+1}\right)+\frac{\varepsilon}{\lambda} B_{X^{*}}, \quad \text { and } \quad \sum_{i=0}^{m+1} x_{i}^{*}=0 .
\end{gathered}
$$

Copyright $@$ by SIAM. Unauthorized reproduction of this article is prohibited. 
It remains to show (4.10). To do this, note from (4.14) that $\sum_{i=0}^{m} x_{i}^{*}=-x_{m+1}^{*}$, and so

$$
\sum_{i=0}^{m+1}\left\|x_{i}^{*}\right\| \leq 2 \sum_{i=0}^{m}\left\|x_{i}^{*}\right\|
$$

Similarly, by (4.14), one has $-y_{k, k}^{*}=y_{m+1, k}^{*}+\sum_{i=0, i \neq k} y_{i, k}^{*}$, and so

$$
\sum_{k=0}^{m}\left\|y_{k, k}^{*}\right\| \leq \sum_{k=0}^{m}\left\|y_{m+1, k}^{*}\right\|+\sum_{i, k=0, i \neq k}^{m}\left\|y_{i, k}^{*}\right\|
$$

hence

$$
\begin{aligned}
\sum_{i=0}^{m+1} \sum_{k=0}^{m}\left\|y_{i, k}^{*}\right\| & \leq \sum_{k=0}^{m}\left\|y_{m+1, k}^{*}\right\|+\sum_{i, k=0, i \neq k}^{m}\left\|y_{i, k}^{*}\right\|+\sum_{k=0}^{m}\left\|y_{k, k}^{*}\right\| \\
& \leq 2\left(\sum_{k=0}^{m}\left\|y_{m+1, k}^{*}\right\|+\sum_{i, k=0, i \neq k}^{m}\left\|y_{i, k}^{*}\right\|\right) .
\end{aligned}
$$

By adding up the estimates (4.18) and (4.19) and making use of (4.13), we have

$$
1 \leq 2\left(\sum_{i=0}^{m}\left\|x_{i}^{*}\right\|+\sum_{k=0}^{m}\left\|y_{m+1, k}^{*}\right\|+\sum_{i, k=0, i \neq k}^{m}\left\|y_{i, k}^{*}\right\|\right)
$$

and so

$$
\begin{aligned}
\frac{1}{2} & \leq \sum_{i=0}^{m}\left(\left\|x_{i}^{*}\right\|+\left\|c_{i}^{*}\right\|\right)+\sum_{k=0}^{m}\left\|y_{m+1, k}^{*}\right\|+\sum_{i, k=0, i \neq k}^{m}\left\|y_{i, k}^{*}-c_{i}^{*}\right\| \\
& <\sum_{i=0}^{m}\left(\left\|x_{i}^{*}\right\|+\left\|c_{i}^{*}\right\|\right)+\frac{\varepsilon}{\lambda}
\end{aligned}
$$

(see (4.17)). Thus the first inequality in (4.10) holds. Moreover, respectively by (4.13) and (4.17), note that

$$
\sum_{i=0}^{m}\left(\left\|x_{i}^{*}\right\|+\left\|y_{m+1, i}^{*}\right\|\right) \leq 1 \quad \text { and } \quad \sum_{i=0}^{m}\left\|c_{i}^{*}-y_{m+1, i}^{*}\right\|<\frac{\varepsilon}{\lambda} .
$$

Thus, by the triangle inequality, we also see that the second inequality in (4.10) holds. The proof is completed.

Theorem 4.4. Let $\Phi_{0}$ be bounded below on the feasible set $Z$ with respect to the ordering cone $K_{0}$, and suppose that $K_{0}$ has a bounded base. Then one of the following two assertions holds:

(i) For any $\varepsilon>0$, there exist $\bar{x} \in Z$ and $\bar{y}_{0} \in \Phi_{0}(\bar{x})$ such that $\left(\bar{x}, \bar{y}_{0}\right)$ is an $\varepsilon$ Pareto solution of (4.1), and there exist $x_{0} \in B(\bar{x}, \varepsilon), y_{0} \in \Phi_{0}\left(x_{0}\right) \cap$ $B\left(\bar{y}_{0}, \varepsilon\right), x_{i} \in B(\bar{x}, \varepsilon)$ and $y_{i} \in \Phi_{i}\left(x_{i}\right) \cap\left(-K_{i}+\varepsilon B_{Y_{i}}\right)(1 \leq i \leq m), a \in A \cap$ $B(\bar{x}, \varepsilon)$, and $c_{i}^{*} \in K_{i}^{+}$satisfying the following properties:

$$
\sum_{i=0}^{m}\left\|c_{i}^{*}\right\|=1 \quad \text { and } \quad 0 \in \sum_{i=0}^{m} D_{c}^{*} \Phi_{i}\left(x_{i}, y_{i}\right)\left(c_{i}^{*}+\varepsilon B_{Y_{i}^{*}}\right)+N_{c}(A, a)+\varepsilon B_{X^{*}} .
$$

Copyright ( by SIAM. Unauthorized reproduction of this article is prohibited. 
(ii) For any $\varepsilon>0$, there exist $\bar{x} \in Z$ and $\bar{y}_{0} \in \Phi_{0}(\bar{x})$ such that $\left(\bar{x}, \bar{y}_{0}\right)$ is an $\varepsilon$ Pareto solution of (4.1) and there exist $x_{0} \in B(\bar{x}, \varepsilon), \quad y_{0} \in \Phi_{0}\left(x_{0}\right) \cap$ $B\left(\bar{y}_{0}, \varepsilon\right), x_{i} \in B(\bar{x}, \varepsilon)$ and $y_{i} \in \Phi_{i}\left(x_{i}\right) \cap\left(-K_{i}+\varepsilon B_{Y_{i}}\right)(1 \leq i \leq m), a \in A \cap$ $B(\bar{x}, \varepsilon)$,

$$
x_{i}^{*} \in D_{c}^{*} \Phi_{i}\left(x_{i}, y_{i}\right)\left(\varepsilon B_{Y_{i}^{*}}\right), \quad \text { and } \quad a^{*} \in N_{c}(A, a)+\varepsilon B_{X^{*}}
$$

satisfying the following properties:

$$
\sum_{i=0}^{m} x_{i}^{*}+a^{*}=0 \quad \text { and } \quad \sum_{i=0}^{m}\left\|x_{i}^{*}\right\|+\left\|a^{*}\right\|=1 .
$$

Proof. By Proposition 4.2, for any $n \in \mathbb{N}$ there exist $\bar{x}_{n} \in Z$ and $\bar{y}_{n} \in \Phi_{0}\left(\bar{x}_{n}\right)$ such that $\left(\bar{x}_{n}, \bar{y}_{n}\right)$ is a $\frac{1}{n^{2}}$-Pareto solution of (4.1). By Theorem 4.3 (applied to $\varepsilon=\frac{1}{n^{2}}$ and $\left.\lambda=\frac{1}{n}\right)$, there exist $x_{0}(n) \in B\left(\bar{x}_{n}, \frac{1}{n}\right), \quad y_{0}(n) \in \Phi_{0}\left(x_{0}(n)\right) \cap B\left(\bar{y}_{n}, \frac{1}{n}\right), \quad x_{i}(n) \in B\left(\bar{x}_{n}, \frac{1}{n}\right)$ and $y_{i}(n) \in \Phi_{i}\left(x_{i}(n)\right) \cap\left(-K_{i}+\frac{1}{n} B_{Y_{i}}\right)(1 \leq i \leq m), x_{m+1}(n) \in A \cap B\left(\bar{x}_{n}, \frac{1}{n}\right), c_{i}^{*}(n) \in$ $K_{i}^{+}$,

$$
x_{i}^{*}(n) \in D_{c}^{*} F\left(x_{i}(n), y_{i}(n)\right)\left(c_{i}^{*}(n)+\frac{1}{n} B_{Y_{i}^{*}}\right)+\frac{1}{n} B_{X^{*}}, \quad 0 \leq i \leq m,
$$

and

$$
x_{m+1}^{*}(n) \in N_{c}\left(A, x_{m+1}(n)\right)+\frac{1}{n} B_{X^{*}}
$$

such that

$$
\sum_{i=0}^{m+1} x_{i}^{*}(n)=0 \quad \text { and } \quad 1+\frac{1}{n}>\max _{0 \leq i \leq m}\left\|x_{i}^{*}(n)\right\|+\left\|c_{i}^{*}(n)\right\|>\frac{1}{2}-\frac{1}{n}
$$

For each $n \in \mathbb{N}$, let $r_{n}:=\sum_{i=0}^{m}\left\|c_{i}^{*}(n)\right\|$. We first consider the case when $\left\{r_{n}\right\}$ is not convergent to 0 . In this case, without loss of generality, we assume that $r_{n} \geq r$ for some positive constant $r$ and for all $n \in \mathbb{N}$ (if necessary take a subsequence). Let $\tilde{c}_{i}^{*}(n):=\frac{c_{i}^{*}(n)}{r_{n}}$. Then, $\tilde{c}_{i}^{*}(n) \in K_{i}^{+}, \sum_{i=0}^{m}\left\|\tilde{c}_{i}^{*}(n)\right\|=1$, and it follows from (4.20)-(4.22) that

$0 \in \sum_{i=0}^{m} D_{c}^{*} F\left(x_{i}(n), y_{i}(n)\right)\left(\tilde{c}_{i}^{*}(n)+\frac{1}{n r} B_{Y_{i}^{*}}\right)+N_{c}\left(A, x_{m+1}(n)\right)+\frac{m+2}{n r} B_{X^{*}} \quad \forall n \in \mathbb{N}$.

This implies that (i) holds. Now assume that $r_{n} \rightarrow 0$. In this case, by (4.22), $l_{n}:=\sum_{i=0}^{m+1}\left\|x_{i}^{*}(n)\right\| \geq \frac{1}{3}$ for all $n$ sufficiently large. It follows from (4.20)-(4.22) that

$$
\begin{gathered}
\frac{x_{i}^{*}(n)}{l_{n}} \in D_{c}^{*} F\left(x_{i}(n), y_{i}(n)\right)\left(\left(\frac{r_{n}}{l_{n}}+\frac{1}{n l_{n}}\right) B_{Y_{i}^{*}}\right)+\frac{1}{n l_{n}} B_{X^{*}}, \quad 0 \leq i \leq m, \\
\frac{x_{m+1}^{*}(n)}{l_{n}} \in N_{c}\left(A, x_{m+1}(n)\right)+\frac{1}{n l_{n}} B_{X^{*}},
\end{gathered}
$$

Copyright ( $\odot$ by SIAM. Unauthorized reproduction of this article is prohibited. 


$$
\sum_{i=0}^{m+1} \frac{x_{i}^{*}(n)}{l_{n}}=0, \quad \text { and } \quad \sum_{i=0}^{m+1}\left\|\frac{x_{i}^{*}(n)}{l_{n}}\right\|=1 .
$$

This implies that (ii) holds. The proof is completed.

In the Asplund space case, similar to the proofs of Theorems 4.3 and 4.4, we can prove the following Theorems 4.5 and 4.6 (but with Theorem 3.4 replacing Theorem 3.1).

Theorem 4.5. Let $X, Y_{0}, \ldots, Y_{m}$ be Asplund spaces. Let $\varepsilon>0$ and $\left(\bar{x}, \bar{y}_{0}\right)$ be a weak $\varepsilon$-Pareto solution of (4.1). Let $\bar{y}_{i} \in \Phi_{i}(\bar{x}) \cap-K_{i}(i=1, \ldots, m)$. Then, for any $\lambda>0$, there exist $x_{i} \in B(\bar{x}, \lambda), y_{i} \in \Phi_{i}\left(x_{i}\right) \cap B\left(\bar{y}_{i}, \lambda\right), x_{m+1} \in A \cap B(\bar{x}, \lambda), c_{i}^{*} \in K_{i}^{+}$,

$$
\begin{aligned}
x_{i}^{*} & \in \hat{D}^{*} \Phi_{i}\left(x_{i}, y_{i}\right)\left(c_{i}^{*}+\frac{\varepsilon}{\lambda} B_{Y_{i}^{*}}\right)+\frac{\varepsilon}{\lambda} B_{X^{*}}, \quad 0 \leq i \leq m, \quad \text { and } \\
x_{m+1}^{*} & \in \hat{N}\left(A, x_{m+1}\right)+\frac{\varepsilon}{\lambda} B_{X^{*}}
\end{aligned}
$$

such that

$$
\sum_{i=0}^{m+1} x_{i}^{*}=0 \quad \text { and } \quad \frac{1}{2}-\frac{\varepsilon}{\lambda}<\sum_{i=0}^{m}\left(\left\|x_{i}^{*}\right\|+\left\|c_{i}^{*}\right\|\right)<1+\frac{\varepsilon}{\lambda}
$$

Theorem 4.6. Let $X, Y_{0}, \ldots, Y_{m}$ be Asplund spaces. Let $\Phi_{0}$ be bounded below on the feasible set $Z$ with respect to the ordering cone $K_{0}$, and suppose that $K_{0}$ has a bounded base. Then, one of the following two assertions holds:

(i) For any $\varepsilon>0$, there exist $\bar{x} \in Z$ and $\bar{y}_{0} \in \Phi_{0}(\bar{x})$ such that $\left(\bar{x}, \bar{y}_{0}\right)$ is an $\varepsilon$ Pareto solution of (4.1) and there exist $x_{0} \in B(\bar{x}, \varepsilon), \quad y_{0} \in \Phi_{0}\left(x_{0}\right) \cap$ $B\left(\bar{y}_{0}, \varepsilon\right), \quad x_{i} \in B(\bar{x}, \varepsilon)$ and $y_{i} \in \Phi_{i}\left(x_{i}\right) \cap\left(-K_{i}+\varepsilon B_{Y_{i}}\right) \quad(1 \leq i \leq m), \quad a \in$ $A \cap B(\bar{x}, \varepsilon)$, and $c_{i}^{*} \in K_{i}^{+}$satisfying the following properties:

$$
\sum_{i=0}^{m}\left\|c_{i}^{*}\right\|=1 \quad \text { and } \quad 0 \in \sum_{i=0}^{m} \hat{D}^{*} \Phi_{i}\left(x_{i}, y_{i}\right)\left(c_{i}^{*}+\varepsilon B_{Y_{i}^{*}}\right)+\hat{N}(A, a)+\varepsilon B_{X^{*}}
$$

(ii) For any $\varepsilon>0$, there exist $\bar{x} \in Z$ and $\bar{y}_{0} \in \Phi_{0}(\bar{x})$ such that $\left(\bar{x}, \bar{y}_{0}\right)$ is an $\varepsilon$ Pareto solution of (4.1) and there exist $x_{0} \in B(\bar{x}, \varepsilon), y_{0} \in \Phi\left(x_{0}\right) \cap B\left(\bar{y}_{0}, \varepsilon\right)$, $x_{i} \in B(\bar{x}, \varepsilon)$ and $y_{i} \in \Phi_{i}\left(x_{i}\right) \cap\left(-K_{i}+\varepsilon B_{Y_{i}}\right)(1 \leq i \leq m), a \in A \cap B(\bar{x}, \varepsilon)$,

$$
x_{i}^{*} \in \hat{D}^{*} \Phi_{i}\left(x_{i}, y_{i}\right)\left(\varepsilon B_{Y_{i}^{*}}\right), \quad \text { and } \quad a^{*} \in \hat{N}(A, a)+\varepsilon B_{X^{*}}
$$

satisfying the following properties:

$$
\sum_{i=0}^{m} x_{i}^{*}+a^{*}=0 \quad \text { and } \quad \sum_{i=0}^{m}\left\|x_{i}^{*}\right\|+\left\|a^{*}\right\|=1 .
$$

Under the Lipschitz assumption, we have the following sharper result.

Theorem 4.7. Let $X, Y_{0}, \ldots, Y_{m}$ be Asplund spaces and $\Phi_{0}$ be bounded below on the feasible set $Z$ with respect to the ordering cone $K_{0}$. Suppose that $K_{0}$ has a bounded base and that each $\Phi_{i}$ is Lipschitz. Then part (i) of Theorem 4.6 holds.

Proof. Let $L>0$ be the Lipschitz constant of each $\Phi_{i}$. Then, by [14, Theorem 3.2], one has

Copyright (C) by SIAM. Unauthorized reproduction of this article is prohibited. 


$$
\sup \left\{\left\|x^{*}\right\|: x^{*} \in \hat{D}^{*} \Phi_{i}\left(x_{i}, y_{i}\right)\left(y_{i}^{*}\right)\right\} \leq L\left\|y_{i}^{*}\right\|
$$

for all $\left(x_{i}, y_{i}\right) \in \operatorname{Gr}\left(\Phi_{i}\right)$ and $y_{i}^{*} \in Y_{i}^{*}$. By Theorem 4.6, we need only show that Theorem 4.6(ii) does not hold for $\varepsilon \in\left(0, \frac{1}{3(m+1) L}\right)$. Let $x_{i} \in X, y_{i} \in \Phi_{i}\left(x_{i}\right), a \in A$, $x_{i}^{*} \in \hat{D}^{*} \Phi_{i}\left(x_{i}, y_{i}\right)\left(\frac{1}{3(m+1) L} B_{Y_{i}^{*}}\right), \quad$ and $\quad a^{*} \in N_{c}(A, a)+\frac{1}{3(m+1) L} B_{X^{*}} \quad$ satisfy $\quad \sum_{i=0}^{m} x_{i}^{*}+$ $a^{*}=0$. It suffices to show that $\sum_{i=0}^{m}\left\|x_{i}^{*}\right\|+\left\|a^{*}\right\| \neq 1$. By (4.23), one has $\left\|x_{i}^{*}\right\| \leq L \frac{1}{3(m+1) L}=\frac{1}{3(m+1)}$. Hence $\quad \sum_{i=0}^{m}\left\|x_{i}^{*}\right\| \leq \frac{1}{3} \quad$ and $\quad\left\|a^{*}\right\|=\left\|\sum_{i=0}^{m} x_{i}^{*}\right\| \leq \frac{1}{3}$. So $\sum_{i=0}^{m}\left\|x_{i}^{*}\right\|+\left\|a^{*}\right\| \leq \frac{2}{3}$. This completes the proof.

Remark. Let $f: X \rightarrow \mathbb{R} \cup\{+\infty\}$ be a proper lower semicontinuous function, and define

$$
F(x)=[f(x),+\infty) \text { and } \quad G(x)=\{f(x)\} \quad \forall x \in X
$$

Recall (cf. [14], [23]) the following known properties:

(i) If $\left(x^{*},-\lambda\right) \in \hat{N}(\operatorname{Gr}(F),(x, t))$, then $\lambda \geq 0$.

(ii) If $\lambda>0$ and $\left(x^{*},-\lambda\right) \in \hat{N}(\operatorname{Gr}(F),(x, t))$, then $t=f(x)$ and $\hat{D}^{*} F(x, f(x))(\lambda)$ $=\lambda \hat{\partial} f(x)$.

(iii) For any $(x, t) \in \operatorname{Gr}(F), \hat{D}^{*} F(x, t)(0)=\hat{D}^{*} F(x, f(x))(0)=\hat{\partial}^{\infty} f(x)$.

(iv) If $f$ is locally Lipschitz, then $\hat{\partial}^{\infty} f(x)=\{0\}$.

(v) For any $\lambda \neq 0, \hat{D}^{*} G(x, f(x))(\lambda)=\hat{\partial}(\lambda f)(x)$.

(vi) $\hat{D}^{*} G(x, f(x))(0)=\hat{\partial}^{\infty} f(x)$.

Let $\phi_{i}: X \rightarrow \mathbb{R}$ be lower semicontinuous functions $(i=0,1, \ldots, m)$, $\Phi_{i}(x)=\left[\phi_{i}(x),+\infty\right)$ for $0 \leq i \leq n$, and $\Phi_{i}(x)=\left\{\phi_{i}(x)\right\}$ for $n<i \leq m$. In the case when $Y_{0}=Y_{1}=\cdots=Y_{m}=\mathbb{R}, K_{0}=\cdots=K_{n}=\mathbb{R}_{+}$, and $K_{n+1}=\cdots=K_{m}=\{0\}$, (4.1) reduces to the usual numerical constraint optimization problem. Thus, in this special case, the coderivatives appearing in Theorems 4.5, 4.6, and 4.7 can be represented in terms of the Fréchet subdifferential of $\Phi_{i}$; in particular, Theorem 4.7 recaptures Mordukhovich and Wang's result mentioned in section 1.

Remark. Let $G: X \rightarrow 2^{Y_{1} \times \cdots \times Y_{m}}$ be such that

$$
G(x):=\Phi_{1}(x) \times \cdots \times \Phi_{m}(x) \quad \forall x \in X
$$

and $K:=K_{1} \times \cdots \times K_{m}$. Then vector optimization problem (4.1) can rewritten as

$$
\begin{aligned}
& K_{0}-\min \Phi_{0}(x), \\
& G(x) \cap-K \neq \varnothing, \\
& x \in A .
\end{aligned}
$$

It is clear that the feasible set of (4.1) and the one of (4.24) are identical. Hence $\left(\bar{x}, \bar{y}_{0}\right)$ is an $\varepsilon$-Pareto solution (resp., a weak $\varepsilon$-Pareto solution) of (4.1) if and only if it is an $\varepsilon$ Pareto solution (resp., a weak $\varepsilon$-Pareto solution) of (4.24). Note that

$$
\sum_{i=1}^{m} \hat{D}^{*} \Phi_{i}\left(x, y_{i}\right)\left(y_{i}^{*}\right) \subset \hat{D}^{*} G\left(x,\left(y_{1}, \ldots, y_{m}\right)\right)\left(y_{1}^{*}, \ldots, y_{m}^{*}\right)
$$

for any $x \in X, y_{i} \in \Phi_{i}(x)$, and $y_{i}^{*} \in Y_{i}^{*}(i=1, \ldots, m)$. But, even in the special case when $\Phi_{i}(x)=\left[\left(\phi_{i}(x),+\infty\right)\right)$ for $1 \leq i \leq n$ and $\Phi_{i}(x)=\left\{\phi_{i}(x)\right\}$ for $n<i \leq m$, one cannot establish the converse inclusion of $(*)$. As for the coderivatives with respect

Copyright (C) by SIAM. Unauthorized reproduction of this article is prohibited. 
to the Clarke normal cones, the relationship between $\sum_{i=1}^{m} D_{c}^{*} \Phi_{i}\left(x, y_{i}\right)\left(y_{i}^{*}\right)$ and $D_{c}^{*} G\left(x,\left(y_{1}, \ldots, y_{m}\right)\right)\left(y_{1}^{*}, \ldots, y_{m}^{*}\right)$ is more complicated; we don't even know whether or not the following inclusion $\left(*^{\prime}\right)$ is true:

$$
\sum_{i=1}^{m} D_{c}^{*} \Phi_{i}\left(x, y_{i}\right)\left(y_{i}^{*}\right) \subset D_{c}^{*} G\left(x,\left(y_{1}, \ldots, y_{m}\right)\right)\left(y_{1}^{*}, \ldots, y_{m}^{*}\right) .
$$

Hence, we cannot establish Theorems 4.3-4.7in terms of the corresponding necessary or sufficient conditions for a weak $\varepsilon$-Pareto solution (or an $\varepsilon$-Pareto solution) of (4.24).

Unfortunately, Theorems 4.3-4.7 cannot cover Chou, Ng, and Pang's result mentioned in section 1 (because the $\varepsilon$-minimizer of $\phi$ over $A$ appearing in their result is itself an " $\varepsilon$-critical point" of $\phi$ over $A$ ). For the rest of this paper, let us consider the following problem (which is a special case of (4.1)):

$$
K_{0}-\min \Phi_{0}(x) \quad \text { subject to } x \in A,
$$

where $\Phi_{0}: X \rightarrow Y_{0}$ is a single-valued function and $A$ is a nonempty closed subset of $X$. The absence of functional constraint would allow us to draw some stronger conclusions and thereby extend the corresponding numerical result of Chou, $\mathrm{Ng}$, and Pang.

For $\varepsilon>0$, we say that $\bar{x} \in A$ is an $\varepsilon$-Pareto solution of (4.25) if

$$
\operatorname{diam}\left(\left(\Phi_{0}(A)+K_{0}\right) \cap\left(\Phi_{0}(\bar{x})-K_{0}\right)\right)<\varepsilon .
$$

Let $\operatorname{epi}_{K_{0}}\left(\Phi_{0}\right)$ denote the epi-gragh of $\Phi_{0}$ with respect to $K_{0}$ and be defined by

$$
\operatorname{epi}_{K_{0}}:=\left\{(x, y) \in X \times Y_{0}: y \in \Phi_{0}(x)+K_{0}\right\} .
$$

Imitating subdifferential formula ( $\mathrm{CF}$ ) of a scalar-valued function, we adopt the following coderivative $D_{e}^{*} \Phi_{0}(x): Y_{0}^{*} \rightrightarrows X^{*}$ defined by

$$
D_{e}^{*} \Phi_{0}(x)\left(y^{*}\right):=\left\{x^{*} \in X^{*}:\left(x^{*},-y^{*}\right) \in N_{c}\left(\operatorname{epi}_{K_{0}}\left(\Phi_{0}\right),\left(x, \Phi_{0}(x)\right)\right)\right\} \quad \forall y^{*} \in Y_{0}^{*} .
$$

Noting that

$$
T_{c}\left(\operatorname{epi}_{K_{0}}\left(\Phi_{0}\right),\left(x, \Phi_{0}(x)\right)\right)=T_{c}\left(\operatorname{epi}_{K_{0}}\left(\Phi_{0}\right),\left(x, \Phi_{0}(x)\right)\right)+\{0\} \times K_{0},
$$

it is easy to verify that

$$
\operatorname{dom}\left(D_{e}^{*} \Phi_{0}(x)\right) \subset K_{0}^{+} .
$$

We will need the following lemma, which is of some interest by itself.

Lemma 4.8. Let $x \in X$ and $y^{*} \in K_{0}^{+}$. Suppose that $\Phi_{0}: X \rightarrow Y_{0}$ is locally Lipschitz. Then

$$
\partial_{c}\left(y^{*} \circ \Phi_{0}\right)(x) \subset D_{e}^{*} \Phi_{0}(x)\left(y^{*}\right) .
$$

Proof. The version holds trivially if $y^{*}=0$. Next assume that $y^{*} \in K_{0}^{+} \backslash\{0\}$. Then, there exists $c_{0} \in K_{0}$ such that $\left\langle y^{*}, c_{0}\right\rangle>0$. Let

$$
S:=\left\{\left(u,\left\langle y^{*}, v\right\rangle\right):(u, v) \in T_{c}\left(\operatorname{epi}_{K_{0}}\left(\Phi_{0}\right),\left(x, \Phi_{0}(x)\right)\right)\right\} .
$$

By $(\mathrm{CF})$, we only need to show that

Copyright ( by SIAM. Unauthorized reproduction of this article is prohibited. 


$$
S \subset T_{c}\left(\operatorname{epi}\left(y^{*} \circ \Phi_{0}\right)(x),\left(x,\left\langle y^{*}, \Phi_{0}(x)\right\rangle\right)\right) .
$$

To do this, let $(u, r) \in S$. Then there exists $v \in Y_{0}$ such that

$$
(u, v) \in T_{c}\left(\operatorname{epi}_{K_{0}}\left(\Phi_{0}\right),\left(x, \Phi_{0}(x)\right)\right) \text { and } r=\left\langle y^{*}, v\right\rangle .
$$

Consider any sequences $\left\{\left(x_{n}, t_{n}\right)\right\} \subset \operatorname{epi}\left(y^{*} \circ \Phi_{0}\right)$ converging to $\left(x,\left\langle y^{*}, \Phi_{0}(x)\right\rangle\right)$ and $\left\{s_{n}\right\} \subset \mathbb{R}_{+}$converging to 0 . It is clear that $\left\{\left(x_{n}, \Phi_{0}\left(x_{n}\right)+\frac{t_{n}-\left\langle y^{*}, \Phi_{0}\left(x_{n}\right)\right\rangle}{\left\langle y^{*}, c_{0}\right\rangle} c_{0}\right)\right\}$ is a sequence in epi $K_{0}\left(\Phi_{0}\right)$ converging to $\left(x, \Phi_{0}(x)\right)$. Hence there exists a sequence $\left\{\left(u_{n}, v_{n}\right)\right\}$ converging to $(u, v)$ such that

$$
\left(x_{n}, \Phi_{0}\left(x_{n}\right)+\frac{t_{n}-\left\langle y^{*}, \Phi_{0}\left(x_{n}\right)\right\rangle}{\left\langle y^{*}, c_{0}\right\rangle} c_{0}\right)+s_{n}\left(u_{n}, v_{n}\right) \in \operatorname{epi}_{K_{0}}\left(\Phi_{0}\right) \quad \forall n \in \mathbb{N} .
$$

This implies that

$$
\left(x_{n}, t_{n}\right)+s_{n}\left(u_{n},\left\langle y^{*}, v_{n}\right\rangle\right) \in \operatorname{epi}\left(y^{*} \circ \Phi_{0}\right) \quad \forall n \in \mathbb{N} .
$$

Since $\left(u_{n},\left\langle y^{*}, v_{n}\right\rangle\right) \rightarrow(u, r)$, it follows that $(u, r) \in T_{c}\left(\operatorname{epi}\left(y^{*} \circ \Phi_{0}\right),\left(x,\left\langle y^{*}, \Phi_{0}(x)\right\rangle\right)\right)$. This shows that (4.26) holds.

In the special case when $\left(Y_{0}, K_{0}\right)=\left(\mathbb{R}, \mathbb{R}_{+}\right)$, the following theorem recaptures Chou, Ng, and Pang's result. For the vector case, we need the condition that $\operatorname{int}\left(K_{0}^{+}\right)$is nonempty. This condition is equivalent to that $K_{0}$ has a bounded base; in this case, $\left\{c \in C:\left\langle y^{*}, c\right\rangle=1\right\}$ is a bounded base of $C$ for any $y^{*} \in \operatorname{int}\left(C^{+}\right)$.

Theorem 4.9. Let $y^{*} \in \operatorname{int}\left(K_{0}^{+}\right)$and suppose that $\Phi_{0}: X \rightarrow Y_{0}$ is a locally Lipschtiz function such that $\Phi_{0}$ is bounded below on $A$ with respect to $K_{0}$. Then, for any $\varepsilon>0$, there exists $x_{\varepsilon} \in A$ such that $x_{\varepsilon}$ is an $\varepsilon$-Pareto solution of (4.25) and

$$
d\left(0, D_{e}^{*} \Phi_{0}\left(x_{\varepsilon}\right)\left(y^{*}\right)+N_{c}\left(A, x_{\varepsilon}\right)\right)<\varepsilon .
$$

Proof. Let $\Theta:=\left\{c \in K_{0}:\left\langle y^{*}, c\right\rangle=1\right\}$. Then $\Theta$ is a bounded base of $K_{0}$. Since $\Phi_{0}$ is bounded below on $A$ with respect to $K_{0}, y^{*} \circ \Phi_{0}$ is bounded below on $A$. By Lemma 4.8 and Chou, Ng, and Pang's result, there exists $x_{\varepsilon} \in A$ such that

$$
\left\langle y^{*}, \Phi_{0}\left(x_{\varepsilon}\right)\right\rangle<\inf _{x \in A}\left\langle y^{*}, \Phi_{0}(x)\right\rangle+\frac{\varepsilon}{M+1}
$$

and (4.27) holds, where $M:=\sup _{\theta \in \Theta}\|\theta\|$. It remains to show that $x_{\varepsilon}$ is an $\varepsilon$-Pareto solution of (4.25). We only need to show that

$$
\left(\Phi_{0}(A)+K_{0}\right) \cap\left(\Phi_{0}\left(x_{\varepsilon}\right)-K_{0}\right) \subset-\left[0, \frac{\varepsilon}{M+1}\right] \Theta+\Phi_{0}\left(x_{\varepsilon}\right)
$$

(as $\operatorname{diam}\left(\left[0, \frac{\varepsilon}{M+1}\right] \Theta\right)<\varepsilon$ ). To do this, let $y=\Phi_{0}\left(x_{\varepsilon}\right)-t \theta \geq_{K_{0}} \Phi_{0}(a)$ for some $t \in[0,+\infty), \theta \in \Theta$, and $a \in A$. Since $y^{*} \in \operatorname{int}\left(K_{0}^{+}\right)$, it follows from (4.28) that

$$
t=\left\langle y^{*}, t \theta\right\rangle \leq\left\langle y^{*}, \Phi_{0}\left(x_{\varepsilon}\right)-\Phi_{0}(a)\right\rangle<\frac{\varepsilon}{M+1} .
$$

Therefore (4.29) is shown.

Acknowledgments. The authors wish to thank the associated editor and referees for their helpful comments.

Copyright ( by SIAM. Unauthorized reproduction of this article is prohibited. 


\section{REFERENCES}

[1] T. Q. BaO And B. S. Mordukhovich, Variational principles for set-valued mappings with applications to multiobjective optimization, Control Cybernet., 36 (2007), pp. 531-562.

[2] T. Q. Bao And B. S. Mordukhovich, Relative Pareto minimizers for multiobjective problems: Existence and optimality conditions, Math. Program., 122 (2010), pp. 301-347.

[3] J. M. Borwein, On the existence of Pareto efficient points, Math. Oper. Res., 8 (1983), pp. 64-73.

[4] C.-C. Chou, K.-F. NG, AND J.-S. PANG, Minimizing and stationary sequences of constrained optimization problems, SIAM J. Control Optim., 36 (1998), pp. 1908-1936.

[5] F. Flores-BaZAn, Ideal, weakly efficient solutions for vector optimzation problems, Math. Program., 93 (2002), pp. 453-475.

[6] A. GötZ AND J. JAhN, The Lagrange multiplier rule in set-valued optimization, SIAM J. Optim., 10 (1999), pp. 331-344.

[7] A. Gopfert, H. Riahi, C. Tamme, and C. Zalinescu, Variational Methods in Partially Ordered Spaces, Springer-Verlag, New York, 2003.

[8] C. Gutiérrez, B. Jimenez, and V. Novo, A unified approach and optimality conditions for approximate solutions of vector optimization problems, SIAM J. Optim., 17 (2006), pp. 688-710.

[9] S. Helbig and D. Pateva, On several concepts for $\varepsilon$-efficiency, OR Spectrum, 16 (1994), pp. 179-186.

[10] J. JAHN, Vector Optimization: Theory, Applications and Extensions, Springer-Verlag, New York, 2004.

[11] G. Jameson, Ordered Linear Spaces, Springer-Verlag, New York, 1970.

[12] G. Y. LI, K. F. NG, AND X. Y. ZHeNG, Unified approach to some geometric results in variational analysis, J. Funct. Anal., 248 (2007), pp. 317-343.

[13] D. T. Luc, Theory of Vector Optimization, Springer-Verlag, New York, 1989.

[14] B. S. Morduknovich, Variational Analysis and Generalized Differentiation, Springer-Verlag, New York, 2006.

[15] B. S. Mordukhovich and Y. Shao, Extremal characterization of Asplund spaces, Proc. Amer. Math. Soc., 124 (1996), pp. 197-205.

[16] B. S. Mordukhovich and Y. Shao, Nonsmooth sequential analysis in Asplund spaces, Trans. Amer. Math. Soc., 348 (1996), pp. 1235-1280.

[17] B. S. Modukhovich, J. S. Treiman, and Q. J. Zhu, An extended extremal principle with applications to multiobjective optimization, SIAM J. Optim., 14 (2003), pp. 359-379.

[18] B. S. Mordukhovich and B. Wang, Necessary suboptimality and optimality conditions via variational principles, SIAM J. Control Optim., 41 (2002), pp. 623-640.

[19] A. B. Nemeth, A nonconvex vector minimization problem, Nonlinear Anal., 10 (1986), pp. 669-678.

[20] H. V. NGai and M. ThÉRA, A fuzzy necessary optimality condition for non-Lipschitz optimization in Asplund spaces, SIAM J. Optim., 12 (2002), pp. 656-668.

[21] Y. Sonntag and C. Zalinescu, Comparison of existence results for efficient points, J. Optim. Theory Appl., 105 (2000), pp. 161-188.

[22] R. T. Rockafellar and R. J.-B. Wets, Variational Analysis, Springer-Verlag, New York, 1998.

[23] W. Sснгтотzeк, Nonsmooth Analysis, Springer-Verlag, New York, 2007.

[24] T. Tanaka, A new approach to approximation of solutions in vector optimization problems, in Proceedings of the Conference of the Association of Asian-Pacific Operational Research Societies, M. Fushimi and K. Tone, eds., Singapore, World Scientific Publishing, River Edge, NJ, 1995, pp. $497-504$.

[25] D. J. White, Epsilon efficiency, J. Optim. Theory Appl., 49 (1986), pp. 319-337.

[26] X. Q. YANG and V. Jeyakumar, First and second-order optimality conditions for convex composite multiobjective optimization, J. Optim. Theory Appl., 95 (1997), pp. 209-224.

[27] J. J. Ye AND Q. J. Zhu, Multiobjective optimization problem with variational inequality constraints, Math. Program., 96 (2003), pp. 139-160.

[28] A. Zaffaroni, Degrees of efficiency and degrees of minimality, SIAM J. Control Optim., 42 (2003), pp. 1071-1086.

[29] X. Y. Zheng And K. F. NG, The Lagrange multiplier rule for multifunctions in Banach spaces, SIAM J. Optim., 17 (2006), pp. 1154-1175.

[30] X. Y. ZHENG AND K. F. NG, Linear regularity for a collection of subsmooth sets in Banach spaces, SIAM J. Optim., 19 (2008), pp. 62-76.

[31] X. Y. Zheng ANd K. F. NG, Calmness for L-subsmooth multifunctions in Banach spaces, SIAM J. Optim., 19 (2009), pp. 1648-1673.

[32] Q. J. ZHU, Hamiltonian necessary conditions for a multiobjective optimal control problem with endpoint constraints, SIAM J. Control Optim., 39 (2000), pp. 97-112.

Copyright @ @ by SIAM. Unauthorized reproduction of this article is prohibited. 